\title{
New level of regulation of MITF activity by direct interaction with RAF proteins
}

\section{Charlène Estrada}

Institut Curie - PSL Research University

\section{Liliana Mirabal-Ortega}

Institut Curie - PSL Research University

\section{Florent Dingli}

Institut Curie - PSL Research University https://orcid.org/0000-0002-7715-2446

\section{Laetitia Besse}

Institut Curie - PSL Research University

\section{Cedric Messaoudi}

Institut Curie - PSL Research University https://orcid.org/0000-0003-3535-7723

\section{Damarys Loew}

Institut Curie - PSL Research University https://orcid.org/0000-0002-9111-8842

\section{Celio Pouponnot}

Institut Curie - PSL Research University

\section{Corine Bertolotto}

Centre Méditerranéen de Médecine Moléculaire

\section{Alain Eychene}

Institut Curie Université Paris Sud https://orcid.org/0000-0002-6818-7225

Sabine Druillennec ( $\nabla$ sabine.druillennec-rodiere@curie.fr)

Institut Curie - INSERM U1021 - CNRS UMR3347 https://orcid.org/0000-0003-0237-1465

\section{Article}

Keywords: MITF activity, RAF proteins, melanoma

Posted Date: April 15th, 2021

DOI: https://doi.org/10.21203/rs.3.rs-404711/v1

License: (1) This work is licensed under a Creative Commons Attribution 4.0 International License. Read Full License 
Version of Record: A version of this preprint was published at Communications Biology on January 28th, 2022. See the published version at https://doi.org/10.1038/s42003-022-03049-w. 


\section{New level of regulation of MITF activity by direct interaction with RAF proteins}

Charlène Estrada ${ }^{1,2,3,4,5}$, Liliana Mirabal-Ortega ${ }^{1,2,3,4,5}$, Florent Dingli ${ }^{6}$, Laetitia Besse ${ }^{7,8}$, Cedric Messaoudi $^{7,8}$, Damarys Loew ${ }^{6}$, Celio Pouponnot ${ }^{1,2,3,4,5}$, Corine Bertolotto ${ }^{9}$, Alain Eychène ${ }^{1,2,3,4,5}$ and Sabine Druillennec ${ }^{1,2,3,4,5 *}$

${ }^{1}$ Institut Curie, Centre de Recherche, Orsay F-91405, France

${ }^{2}$ INSERM U1021, Centre Universitaire, Orsay F-91405, France

${ }^{3}$ CNRS UMR 3347, Centre Universitaire, Orsay F-91405, France

${ }^{4}$ Université Paris-Saclay, Orsay F-91405, France

${ }^{5}$ PSL Research University, Paris F-75006, France

${ }^{6}$ Institut Curie, PSL Research University, Centre de Recherche, Laboratoire de Spectrométrie de Masse Protéomique, 26 rue d'Ulm, Paris 75248 Cedex 05, France

${ }^{7}$ Institut Curie, PSL Research University, CNRS UMS 2016, F-91401 Orsay, France

${ }^{8}$ Université Paris-Saclay, INSERM US43, F-91401 Orsay, France

${ }^{9}$ Université Côte d'Azur, INSERM U1065, Centre Méditerranéen de Médecine Moléculaire (C3M), Nice, France.

Running title: Control of MITF activity by RAF binding

Correspondence:

Dr. Sabine Druillennec e-mail: sabine.druillennec-rodiere@curie.fr

Institut Curie - Centre de Recherche

Centre Universitaire, Bâtiment 110, Rue Henri Becquerel

CS 90030, 91401 ORSAY cedex, France

Tel. 33-1 69863041

Fax. 33-1 69863051

Keywords: ARAF / BRAF / CRAF / melanoma / MITF 


\section{ABSTRACT}

The MITF transcription factor and the RAS/RAF/MEK/ERK pathway are two interconnected main players in melanoma. Understanding how MITF activity is regulated represents a key question since its dynamic modulation is involved in the phenotypic plasticity of melanoma cells and their resistance to therapy. By investigating the role of ARAF in NRAS-driven melanoma through mass spectrometry experiments followed by a functional siRNA-based screen, we unexpectedly identified MITF as a direct ARAF partner. Interestingly, this interaction is conserved among the RAF protein kinase family since the formation of BRAF/MITF and CRAF/MITF complexes was also observed in the cytosol of NRASmutated melanoma cells. The interaction occurs through the kinase domain of RAF proteins and is correlated with their kinase activity level. RAF/MITF complexes modulate MITF nuclear localization by inducing an accumulation of MITF in the cytoplasm, thus negatively controlling its transcriptional activity. Taken together, our study highlights a new level of regulation between two major mediators of melanoma progression, MITF and the MAPK/ERK pathway, which appears more complex than previously anticipated. 


\section{INTRODUCTION}

Cutaneous melanoma is an aggressive tumour arising from malignant transformation of melanocytes

1. The RAS/RAF/MEK/ERK is a key signaling pathway frequently mutated in cutaneous melanoma since activating mutations in either NRAS or BRAF genes occur in $15-20 \%$ and $40-50 \%$ of cases, respectively, the two main mutations being NRAS ${ }^{\mathrm{Q} 61 \mathrm{~K}}$ and $\mathrm{BRAF}^{\mathrm{V} 600 \mathrm{E} 2,3}$. RAS is a GTPase activated via membrane-bound receptors upon stimulation by growth factors. In its GTP-bound form, RAS recruits effectors at the membrane and stimulates a number of downstream intracellular signaling pathways, including the MAPK/ERK pathway ${ }^{4}$. The three RAF serine-threonine kinases, ARAF, BRAF and CRAF conserved in vertebrates are among the main RAS effectors. RAF activation enables subsequent activation by phosphorylation of MEK1 and MEK2, which in turn activate ERK1 and ERK2 ${ }^{5}$. Once translocated in the nucleus, ERK regulates a wide variety of transcription programs leading to modulation of key biological processes such as cell proliferation, survival, migration or differentiation 6.

Using conditional knockout of BRAF and/or CRAF in a mouse melanoma model induced by $\mathrm{NRAS}^{\mathrm{Q} 61 \mathrm{~K}}$, we showed that while BRAF is required downstream of activated NRAS for tumour initiation, both BRAF and CRAF play compensatory functions during late phases of melanomagenesis, thus highlighting the addiction of melanoma to the RAF/ERK pathway ${ }^{7}$. Interestingly, we demonstrated that in the absence of BRAF and CRAF, ARAF alone can sustain both ERK activation and proliferation in NRAS-mutated melanoma cells. In this context, we also observed that ARAF homodimers are sufficient to induce ERK paradoxical activation by vemurafenib, a widely used $\mathrm{BRAF}^{\mathrm{V} 600 \mathrm{E}}$ inhibitor, in the absence of both BRAF and CRAF. Our results suggested a dependency toward ARAF kinase, as well as a possible role of ARAF in resistance mechanisms in cutaneous melanoma. The potential role of ARAF in NRAS-induced melanoma was further strengthened by an in silico search in public databases that allowed to identify patients with metastatic melanomas harbouring an ARAF mutation associated with activating NRAS mutations ${ }^{7}$. Nevertheless, ARAF remains the less studied member of the RAF family because: i) ARAF displays the lowest kinase activity towards MEK compared to other 
RAF proteins ${ }^{8}$, ii) in most cellular models, the role of ARAF is hidden by the predominant roles of BRAF and CRAF.

Microphtalmia-associated transcription factor (MITF) is a master regulator of the melanocytic lineage since it is essential for the differentiation, survival and proliferation of melanocytes ${ }^{9,10}$. MITF belongs to the MiT family, gathering bHLH-LZ domain transcription factors (TFEB, TFEC and TFE3), that can homo- or hetero-dimerize to regulate gene expression ${ }^{11}$. Expressed in about $80 \%$ of human melanoma ${ }^{12,13}$, MITF displays a central regulatory role in melanoma cell phenotypic plasticity. A proposed rheostat model suggests that the global level of MITF activity correlates with the phenotype of melanoma cells: at high levels of activity, MITF sustains the proliferative state of melanoma cells while at lower levels, MITF is associated with an invasive and stem-like phenotype ${ }^{14-17}$. This model has been recently refined with six different states some being resistant to therapy but still on the basis of MITF activity ${ }^{18,19}$. In line with its central role, MITF is finely regulated to ensure the homeostasis of melanocytes or melanoma cells ${ }^{10}$. Among its numerous post-translational regulators, MITF is regulated by ERK2, that phosphorylates the S73 residue inducing both proteasome-mediated degradation and increased activity via the recruitment of $\mathrm{p} 300 / \mathrm{CBP}$ transcription cofactor ${ }^{20-24}$. Altogether, the posttranslational regulation of MITF by ERK pathway has opposite consequences regarding MITF activity, depending on cellular context ${ }^{13}$.

To better characterize the role of ARAF in NRAS-driven melanoma, we searched for new ARAF interactors by mass spectrometry. Our results showed that ARAF directly binds to the transcription factor MITF. ARAF/MITF complexes were found in the cytosol of NRAS-mutated melanoma cells. Not only ARAF, but also BRAF and CRAF interacted with MITF and the kinase active status was required to allow complex formation. At the functional level, RAF/MITF interaction modulates MITF nuclear localization, thus regulating its transcriptional activity. Taken together, these results highlight a new level of regulation of MITF by RAF, two key players of melanoma biology. 


\section{RESULTS and DISCUSSION}

\section{Identification of new ARAF partners by large-scale analysis}

Although our knowledge of ARAF kinase has enlarged over the last decade ${ }^{25}$, ARAF remains understudied compared to the other members of RAF family. Owing to the redundant roles and the high homology of RAF kinases as well as the weak kinase activity of ARAF compared to BRAF and CRAF, it is challenging to study ARAF function in most cellular models where BRAF and CRAF are also expressed. In addition, attribution of a specific function to each RAF kinase is further hampered by their propensity to heterodimerize, especially when looking for binding partners. In the present study, we took advantage of a genetically engineered NRAS-driven melanoma mouse model allowing concomitant ablation of BRAF and CRAF to investigate the role of ARAF. Tumor cells derived from these mice constitute a well-adapted model to study ARAF function in the melanoma context in absence of BRAF and CRAF expression (ARAF-only cells) ${ }^{7}$. The ARAF interactome was established by immunoprecipitation of the endogenous ARAF protein from ARAF-only or control cells followed by analysis of the immune complexes by mass spectrometry in label-free conditions (Fig. 1). ARAF-only cells, which emerged after braf and craf genes ablation in melanoma cultures established from primary NRAS-induced tumours, are highly dependent on ARAF expression for their growth and survival ${ }^{7}$. Control cells display normal levels of BRAF and CRAF, but express low level of ARAF shRNAmediated knockdown, thus allowing relative quantification of the data. The distribution of the 2700 ARAF-interacting proteins in ARAF-only or control cells is illustrated by the volcano plot (Fig. 1a, Table 1). Well-known ARAF partners were found enriched in ARAF-only cells, such as its upstream activator, the GTPase NRAS, or its substrate MEK1, indicating the reliability of the experimental approach (Table 1). In order to identify ARAF relevant partners, which functionally impact melanoma cell proliferation, we developed a siRNA-based functional screen on 99 selected targets (Fig. 1a). These 99 interactors were selected as followed: 69 were chosen among 359 proteins enriched in ARAF-only cells with the following parameters: number of peptides $>9$, ratio $>2$ and adjusted p-value $<0.001$ (Fig. 1a). 15 were from the 72 proteins exclusively identified in ARAF-only cells. We also included 15 
proteins that were found both in our current dataset and in the ARAF interactome published by Zhang et al. ${ }^{26}$. ARAF-only cells growth was followed upon knock-down of the selected partners by siRNA pools transfection (Supplementary Fig. 1). Among the 99 partners tested, 16 impacted the growth of ARAF only melanoma cells. It appeared that 11 ARAF partners had an anti-proliferative effect while 5 proteins were pro-proliferative (labelled in green and red, respectively in Fig. 1b). Among the 132 interactors identified in the ARAF proteome by Zhang et al. ${ }^{26,27}, 107$ were commonly found in our dataset, showing the robustness of the approach. Twenty-four of the common identified partners were included in our screen: 9 were selected by the previously described parameters and 15 additional were chosen after bibliographic analysis. It should be noticed that Zhang et al. have validated their interactome by coimmunoprecipitation experiments on 12 out of 13 randomly selected ARAF interacting proteins with different functions. Eight of these confirmed ARAF partners were tested in the siRNA-based screen and three appear to play a functional role in ARAF only cells: NCL, PARP and PSMC2.

Among the 16 partners that impact melanoma proliferation, we decided to focus on MITF since it represents a key transcription factor for melanoma progression that can be involved in therapy-resistance mechanism. It is also well known to be regulated by the MAPK/ERK pathway ${ }^{10,13}$. Of note, the ARAF interactome by Zhang et al. could not identify MITF as an ARAF partner since it was performed on a heterologous model overexpressing tagged ARAF in HEK293 cells that do not express MITF ${ }^{26,27}$. We next confirmed the pro-proliferative effect of MITF in ARAF-only cells by using two distinct siRNA against MITF in comparison to control siRNA. Since we previously demonstrated that ARAF-only cells rely on ARAF for their proliferation, we included a siRNA targeting ARAF as a positive control (Fig. 1c). Both siRNA against MITF decreased the growth of ARAF-only melanoma cells. Moreover, we observed a good correlation between the effect on cell proliferation and the level of extinction of MITF expression induced by the different siRNA (Fig. 1d), demonstrating that MITF is required for ARAFonly cells growth. 


\section{ARAF directly interacts with MITF}

The interaction between ARAF and MITF was confirmed by direct coimmunoprecipitation experiments of endogenous proteins in ARAF-only cells (Fig. 2a). As shown in Fig. 2b, ARAF/MITF complexes were also detected by Proximity Ligation Assay (PLA) in ARAF-only cells, further revealing that this interaction occurred in the cytoplasm of melanoma cells. Importantly, this interaction appeared to be direct since complex formation was observed between ARAF and MITF human purified recombinant proteins, in an in vitro coimmunoprecipitation assay (Fig. 2c).

\section{Characterization of the RAF/MITF interaction}

While the connection between the ERK/MAPK pathway and MITF is well established in melanoma ${ }^{13}$, a direct interaction between RAF kinases and MITF has never been previously demonstrated. To further substantiate this observation, we tested whether this interaction was specific of ARAF or shared by all the RAF kinases. HEK293T cells were cotransfected with MITF and each of the three different HAtagged RAF proteins. Anti-HA1 immune complexes were then probed with an anti-MITF antibody. Interestingly, we observed that MITF could interact not only with ARAF but also with BRAF and CRAF, the two others members of the RAF family (Fig. 3a-b-c). We confirmed the existence of cytoplasmic BRAF/MITF and CRAF/MITF endogenous complexes by PLA experiments in NRASmutated murine melanoma cells as observed for ARAF (Supplementary Fig. 2). This is the first evidence of a direct interaction between a RAF kinase and MITF, two key players in melanoma cell biology. Although an MITF interactome has been previously reported, RAF kinases were not identified in this study since the authors focused specifically on nuclear interactors by performing nuclear purification ${ }^{28}$. Since the MAPK/ERK pathway is dysregulated by NRAS, but also BRAF mutations in melanoma, we investigated the ability of MITF to interact with the constitutively active $\mathrm{BRAF}^{\mathrm{V} 600 \mathrm{E}}$ mutant. This is the most frequent BRAF mutation in human cancers, which is highly prevalent in melanoma and which markedly increases BRAF kinase activity ${ }^{29}$ (Fig. 3d). We observed that MITF strongly interacts with $\mathrm{BRAF}{ }^{\mathrm{V} 600 \mathrm{E}}$ with an increased affinity compared to wild-type BRAF. To evaluate the requirement of the RAF kinase activity, we also tested the interaction with the BRAF ${ }^{\mathrm{K} 499 \mathrm{M}}$ kinasedead mutant $\left(\mathrm{BRAF}^{\mathrm{KD}}\right)$ containing a Lys-to-Met substitution in its kinase domain (Fig. 3d). In contrast 
to $\mathrm{BRAF}^{\mathrm{V} 600 \mathrm{E}}$, the capacity of $\mathrm{BRAF}^{\mathrm{KD}}$ to bind to MITF was decreased as compared to wild-type BRAF. Therefore, the strength of the binding directly correlates with the activation state of the RAF proteins since MITF strongly interacts with the activated mutant of BRAF, and much less with the BRAF kinasedead mutant. These results suggest that not only an active form of the RAF kinase is required to allow the interaction with MITF, but also that the MITF/BRAF complex formation can occur in a BRAFmutated context.

We next investigated the role of the different domains of BRAF in the interaction with MITF, by using truncated forms of the protein (Fig. 3e). HEK293T cells were cotransfected with plasmids encoding MITF and either the C-terminus or N-terminus part of BRAF, or both. Of note, it was previously demonstrated that the $\mathrm{N}$-terminus regulatory domain of RAF proteins binds to their $\mathrm{C}$-terminus kinase domain in order to regulate their activity ${ }^{30}$. Accordingly, $\mathrm{N}$ - and C-terminus parts co-precipitated when co-expressed (Fig. 3e). Following C-terminus immunoprecipitation in the absence of the N-terminus, a strong interaction with MITF was observed indicating that the N-terminal part of BRAF is not required for MITF binding. Moreover, in these conditions, the presence of the $\mathrm{N}$-terminus did not strengthen the interaction between MITF and the C-terminus (Fig. 3e, right panel). On the opposite, a weak interaction with MITF was seen when the N-terminal domain was immunoprecipitated in the absence of the Cterminus (Fig. 3e, left panel). However, complex formation between the N-terminus and MITF was strongly increased in the presence of the C-terminal part suggesting that, in this condition, the Nterminus does not interact directly with MITF but through the C-terminal domain. Altogether, the results indicate that complex formation with MITF involves the C-terminus region of RAF proteins that contains the kinase domain. These observations also suggest the requirement of a functional kinase domain to stabilize the interaction between RAF and MITF.

\section{Functional role of RAF/MITF interaction}

We next investigated how the MITF/RAF complex formation could affect the respective subcellular localization of each partner, knowing that RAF kinases are cytosolic, whereas MITF can shuttle between the cytosol and the nucleus. HEK293T cells were transfected with epitope-tagged MITF and ARAF, BRAF or CRAF and subcellular localization was analysed by immunofluorescence (Fig. 4). When 
expressed alone, MITF was mainly nuclear while RAF proteins displayed a clear cytoplasmic localization. However, when co-expressed, a relocalization of MITF from nucleus to cytoplasm was observed, indicating that complexes between MITF and ARAF, BRAF or CRAF, are cytoplasmic in agreement with previous observations in PLA experiments (Fig. 2b, Supplementary Fig. 2). This suggests that RAF proteins may retain MITF in the cytoplasm.

In order to better understand the functional consequence of this cytoplasmic interaction, we next investigated how RAF proteins could affect MITF transcriptional activity. HEK293T cells were transfected by constructs encoding a luciferase reporter gene under the control of the MITF-regulated tyrosinase promoter, together with increasing amounts of plasmids encoding the RAF proteins. We found that RAF kinases overexpression led to a decrease in MITF transcriptional activity, in a dosedependent manner. Both BRAF and CRAF overexpression strongly suppressed MITF transcriptional activity while ARAF, which possesses a weaker kinase activity, reduces MITF activity to a lesser extent (Fig. 5). Thus, the inhibition of MITF transcriptional activity by RAF proteins appears to be correlated with their kinase activity. Accordingly, the luciferase activity was strongly decreased by BRAF ${ }^{\mathrm{V} 600 \mathrm{E}}$ mutant as compared to wild-type BRAF while the BRAF kinase-dead mutant had no effect on MITF transcriptional activity. These results showed that binding to RAF kinases negatively regulates MITF transcriptional activity. Taken together, these observations suggest a link between the level of activation of RAF proteins, their ability to form complex with MITF and inhibition of MITF transcriptional activity.

MITF plays a critical role in melanoma cells homeostasis, acting as a master regulator of transcription of numerous target genes involved in a large panel of biological functions (proliferation, cell cycle control, survival, invasion, DNA repair, metabolism, autophagy, etc) ${ }^{14,31-39}$. The contradictory observations regarding the role of MITF in proliferation lead to the establishment of the MITF rheostat model whereby MITF activity is linked to melanoma cell phenotype: in this model, high levels of MITF are associated with pro-proliferative phenotype whereas lower levels are correlated with invasiveness 14,33. Recently, the MITF rheostat model was refined by incorporating six phenotypic states ranging from hyper- to under-differentiated, and associated with different level of MITF activity ${ }^{19}$. The modulation of MITF activity in melanoma cells is highly complex and partly due to a dynamic regulation at 
transcriptional and post-translational levels ${ }^{10}$. Here, we revealed an unsuspected mechanism of MITF activity modulation by demonstrating a direct interaction between RAF kinases and MITF. Indeed, overexpression of ARAF, BRAF or CRAF kinases triggers a partial subcellular relocalization of MITF in the cytoplasm, thus enabling to reduce nuclear concentrations of MITF that could fine tune MITF activity during phenotype switching. Our findings are also in agreement with the study by Ngeow et al. demonstrating that MITF cytoplasmic/nuclear shuttling is controlled by ERK-mediated MITF S73 phosphorylation ${ }^{24}$. Therefore, RAF proteins could act as scaffolds in order to enable the phosphorylation by ERK, thus preventing the nuclear import of MITF and regulating its transcriptional activity. Moreover, since the strength of BRAF/MITF complexes is correlated with the level of BRAF kinase activity, this mechanism of proliferation regulation could happen more particularly when the proproliferative ERK/MAPK pathway is highly activated. Even if the biological significance of the interaction between MITF and RAF kinases deserves further investigations, our study reveals that the regulation of MITF activity by the MAPK/ERK pathway appears more complex than previously anticipated. 


\section{MATERIAL AND METHODS}

Cell lines. ARAF-only or control cells were obtained from previously described NRAS-mutated murine melanoma ${ }^{7}$. ARAF-only cells are double knockout for BRAF and CRAF. Control cells display normal levels of BRAF and CRAF and stably express a shRNA against ARAF (TRCN0000294819, Mission shRNA library, Sigma). Cells were cultured in HAM F-12 Medium (Gibco) containing 10\% foetal bovine serum (FBS), $100 \mathrm{mg} / \mathrm{mL}$ streptomycin, $100 \mathrm{U} / \mathrm{mL}$ penicillin and $2 \mathrm{mM}$ L-glutamine (Invitrogen). Human embryonic kidney 293T (HEK293T) cells were maintained in Dulbecco's modified Eagle's medium (DMEM, Gibco) supplemented with 10\% FBS, $100 \mathrm{mg} / \mathrm{mL}$ streptomycin, $100 \mathrm{U} / \mathrm{mL}$ penicillin, and $1 \mathrm{mg} / \mathrm{mL}$ amphotericin B. Cells were tested for mycoplasma contamination and cultured at $37^{\circ} \mathrm{C}$ and $5 \% \mathrm{CO}_{2}$.

Proteomics and Mass Spectrometry Analysis. ARAF-only or control cells were lysed in NP40 buffer (Tris $\mathrm{pH} 7.5,50 \mathrm{mM}, \mathrm{NaCl}, 150 \mathrm{mM}, 0.5 \% \mathrm{NP} 40$, protease and phosphatase inhibitors). Five or four biological replicates were prepared for each condition, respectively. Endogenous ARAF was immunoprecipitated with ARAF antibody (\#75804, Cell Signaling) and Pierce ${ }^{\mathrm{TM}}$ protein-A magnetic beads on $1 \mathrm{mg}$ of total protein extracts. Immunoprecipitation was performed at $4{ }^{\circ} \mathrm{C}$ for 4 hours and pellets were washed 3 times in NP40 buffer and twice in $100 \mu \mathrm{L}$ of $\mathrm{ABC}$ buffer $\left(25 \mathrm{mM} \mathrm{NH}_{4} \mathrm{HCO}_{3}\right)$. Beads were resuspended in $\mathrm{ABC}$ buffer and digested with $0.20 \mu \mathrm{g}$ of trypsine/LysC (Promega) for 1 hour at $37^{\circ} \mathrm{C}$. Samples were loaded onto homemade Tips packed with Empore ${ }^{\mathrm{TM}} \mathrm{C} 18$ Extraction Disks (3M $\mathrm{M}^{\mathrm{TM}}$ Discs 2215) for desalting. Peptides were eluted using $40 / 60 \mathrm{MeCN} / \mathrm{H}_{2} \mathrm{O}+0.1 \%$ formic acid and vacuum concentrated to dryness. Liquid chromatography was performed with an RSLCnano system (Ultimate 3000, Thermo Scientific) coupled to a Q Exactive HF-X with a Nanospay Flex ion source (Thermo Scientific). Peptides were trapped on a C18 column $(75 \mu \mathrm{m}$ inner diameter $\times 2 \mathrm{~cm}$; nanoViper Acclaim PepMapTM 100, Thermo Scientific) with buffer A (2/98 MeCN/H2O in $0.1 \%$ formic acid) at a flow rate of $2.5 \mu \mathrm{L} / \mathrm{min}$ over $4 \mathrm{~min}$. Separation was performed on a $50 \mathrm{~cm}$ x $75 \mu \mathrm{m}$ C18 column (nanoViper Acclaim PepMapTM RSLC, $2 \mu \mathrm{m}, 100 \AA$, Thermo Scientific) regulated at $50{ }^{\circ} \mathrm{C}$ with a linear gradient of $2 \%$ to $30 \%$ buffer $\mathrm{B}(100 \% \mathrm{MeCN}$ in $0.1 \%$ formic acid) at a flow rate of $300 \mathrm{~nL} / \mathrm{min}$ over $91 \mathrm{~min}$. MS full scans were performed in the ultrahigh-field Orbitrap mass analyzer in ranges $\mathrm{m} / \mathrm{z}$ 
$375-1,500$ with a resolution of 120,000 at $\mathrm{m} / \mathrm{z} 200$. The top 20 intense ions were subjected to Orbitrap via high energy collision dissociation (HCD) activation and a resolution of 15000 with the AGC target set to $10^{5}$ ions. We selected ions with charge state from $2+$ to $6+$ for screening. Normalized collision energy (NCE) was set at 27 and the dynamic exclusion of 40s. For identification, the data were searched against the Mus Musculus UniProt canonical database (22082017 containing 16888 sequences) using Sequest-HT through proteome discoverer (version 2.0). Enzyme specificity was set to trypsin and a maximum of two-missed cleavage sites were allowed. Oxidized methionine carbamidomethyl cysteines and $\mathrm{N}$-terminal acetylation were set as variable modifications. Maximum allowed mass deviation was set to $10 \mathrm{ppm}$ for monoisotopic precursor ions and 0.02 Da for MS/MS peaks. The resulting files were further processed using myProMS v3.5 ${ }^{40}$. FDR calculation used Percolator ${ }^{41}$ and was set to $1 \%$ at the peptide level for the whole study. The label free quantification was performed by peptide Extracted Ion Chromatograms (XICs) computed with MassChroQ version 2.0.1 ${ }^{42}$. For protein quantification, XICs from proteotypic peptides shared between compared conditions (TopN matching), missed cleavages and carbamidomethyl cysteine modified peptides were used. Global MAD normalization was applied on the total signal to correct the XICs for each biological replicate. To estimate the significance of the change in protein abundance, a linear model (adjusted on peptides and biological replicates) based on two-tailed T-tests was performed and p-values were adjusted with a Benjamini-Hochberg FDR. Protein with at least nine total peptides in all replicates, a 2 -fold enrichment and an adjusted p-value $<0.001$ were considered significantly enriched in sample comparison. Unique proteins were considered with at least four total peptides in all replicates.

siRNA-based functional screen. ARAF-only cells were seeded in 96 -wells plate at $8.10^{3}$ cells per well in HAM F-12 Medium without antibiotics. After 24 hours, cells were transfected with siRNA against the 99 putative partners of ARAF selected for the screen (mouse ON-TARGETplus siRNA, pool of 4 siRNA, Dharmacon) or siCTL (ON-TARGETplus non-targeting siRNA, pool of 4 siRNA, Dharmacon) by using DharmaFECT 3 transfection reagent. After 8 hours, medium was changed and the proliferation was followed for 72 hours by using IncuCyte ${ }^{\circledR}$. SiRNA targeting ARAF was a pool of 4 siRNA (J042948-05, J-042948-06, J-042948-07, J-042948-08, Dharmacon). Individual siRNA targeting MITF were from Dharmacon (si mitf\#1 J-047441-05, si mitf\#2 J-047441-07). 
Transfection and coimmunoprecipitation. HEK293T were transfected with pcDNA3-MITF (HAMITF or myc-MITF, gift from C.Bertolotto) and pcDNA3-RAF plasmids (HA-ARAF, HA-BRAF, HACRAF, myc-BRAF, myc-BRAF ${ }^{\mathrm{V} 600 \mathrm{E}}$ or myc-BRAF ${ }^{\mathrm{KD}}$ ) or pcDNA3 empty vector using Lipofectamine reagent (Invitrogen). In cotransfection experiments with $\mathrm{N}$-terminal or C-terminal part of BRAF, either pcDNA3/HA-Nter-BRAF and/or pcDNA3/flag-Cter-BRAF ${ }^{30}$ were transfected with pcDNA3/mycMITF or pcDNA3 empty vectors. After 48 hours, cells were lysed in NP40 buffer and extracts were precipitated overnight at $4^{\circ} \mathrm{C}$ either with anti-HA (3F10, Roche), anti-Flag (M2, Sigma) or anti-Myc (9E10, Santa Cruz) and Pierce ${ }^{\mathrm{TM}}$ protein-G magnetic beads. Immunoprecipitates were washed with NP40 Buffer and boiled in Laemmli's sample buffer. They were then resolved by sodium dodecyl sulfate-polyacrylamide gel electrophoresis and transferred onto a polyvinylidene difluoride membrane (Millipore). For endogenous immunoprecipitation, cell lysates were incubated overnight at $4^{\circ} \mathrm{C}$ with anti-ARAF antibody (\#4432, Cell Signaling) and Pierce ${ }^{\mathrm{TM}}$ protein-A magnetic beads. For immunoprecipitation of recombinant proteins, $15 \mathrm{ng}$ of human recombinant MITF protein (Origene) were incubated overnight at $4{ }^{\circ} \mathrm{C}$ in NP40 Buffer with $75 \mathrm{ng}$ of human recombinant flag-ARAF (Origene) and anti-flag magnetic beads (M2, Sigma).

Western blotting and antibodies. For SDS-PAGE analysis, the membranes were blocked with $5 \%$ milk in PBS Tween 20 (10\%) for $30 \mathrm{~min}$ at room temperature. Membranes were then probed overnight at $4^{\circ} \mathrm{C}$ with the appropriated primary antibodies: anti-MITF (HPA003259, Sigma), anti-HA (3F10, Roche), anti-myc (9E10, Santa Cruz), anti-flag (M2, Sigma), anti-ARAF (\#4432, Cell Signaling) and anti- $\beta$-actin (A1978, Sigma) antibodies. Antigen-antibody complexes were detected by horseradish peroxidase-coupled secondary antibodies followed by enhanced chemiluminescence. Signals were acquired using a cooled-CDD camera (Fusion FX Spectra, Vilber).

Proximity Ligation Assay (PLA). Cells were grown on glass coverslips, fixed and permeabilized. PLA (Duolink) was performed according to the manufacturer's instructions (Sigma) using antibodies against ARAF (cs4432, Cell Signaling) and MITF (ab12039, abcam), BRAF (F7, Santa Cruz) and MITF (HPA003259, Sigma), or CRAF (\#610151, BD Biosciences) and MITF (HPA003259, Sigma). Images were captured using a 3D Leica fluorescent microscope. The average number of dots per cell (identified 
by its nucleus stained with DAPI) was determined by analysing at least 200 different cells with Image $\mathrm{J}$ software.

Immunofluorescence. HEK293T cells were seeded at $3.10^{5}$ cells per well in 6-wells slides (Millicell, Millipore) precoated with poly-L-lysine (Sigma) and transfected with pcDNA3/myc-MITF and pcDNA3/HA-ARAF, pcDNA3/HA-BRAF, pcDNA3/HA-CRAF plasmids or pcDNA3 empty vector as previously described. After 48 hours, cells were fixed in $4 \%$ paraformaldehyde, blocked $(0.1 \%$ Triton $\mathrm{X}-100,10 \%$ goat serum in PBS) and stained overnight at $4{ }^{\circ} \mathrm{C}$ with anti-myc antibody (Santa Cruz) and anti-ARAF (Cell Signaling) or anti-MITF (Sigma) and anti-BRAF (Santa Cruz) or anti-CRAF (BD Biosciences). Anti-mouse Alexa Fluor 594 and anti-rabbit Alexa Fluor 488 or anti-rabbit Alexa Fluor 594 and anti-mouse Alexa Fluor 488 were used for detection. Fluoroshield with DAPI (Sigma) was used as mounting medium. Images were captured using a 3D/optigrid Leica fluorescent microscope. For quantification, the nuclear and cytosolic compartments were selected by applying an automatic threshold (Li Dark method) to the DAPI and FITC images. The nucleus-cytoplasm ratio was then computed by dividing the mean intensity of TexasRed2 (MITF) fluorescence extracted from nucleus region by the mean intensity from cytosolic regions obtained by subtracting DAPI from the FITC surface. The background intensity was measured on each TexasRed 2 image and subtracted from the mean intensities before calculating the ratio.

Luciferase reporter assay. HEK293T cells seeded at $10^{4}$ cells per well in 96-wells plate were cotransfected by using $0.3 \mu \mathrm{L}$ of lipofectamine in a final volume of $100 \mu \mathrm{L}$ with $50 \mathrm{ng}$ of the pTYR-Luc luciferase reporter plasmid (kindly provided by C.Bertolotto), $1.7 \mathrm{ng}$ of pßactin- $\beta$ gal, $5 \mathrm{ng}$ of pcDNA3/myc-MITF and from 0 to $25 \mathrm{ng}$ of pcDNA3 expression vector, empty or containing the RAF coding sequences. Dual luciferase and $\beta$-galactosidase reporter assay was performed 48 hours after transfection, using Dual-Light ${ }^{\mathrm{TM}}$ Luciferase \& $\beta$-Galactosidase Reporter Gene Assay System (Invitrogen). Cells were washed with saline phosphate buffer and lysed with $15 \mu \mathrm{L} /$ well of Dual-Light ${ }^{\mathrm{TM}}$ lysis solution. After 10 min incubation at room temperature, $25 \mu \mathrm{L} /$ well of Buffer A were added and the luciferase activity was measured for 1 second using luminometer ( $\operatorname{TriStar}^{2}$, Berthold) after injection of $100 \mu \mathrm{L} /$ well of Galacton-Plus ${ }^{\circledR}$ diluted 1:100 in Buffer B. After $1 \mathrm{~h}$ incubation in the dark, the $\beta$ - 
galactosidase activity was measured after injection of $100 \mu \mathrm{L} /$ well of Accelerator-II reagent for 0.5 s/well.

Statistics and reproducibility. All statistical analyses were conducted using GraphPad Prism. Each assay was conducted in at least three biological replicates. The exact sample size is given in the legend of each figure. The mean \pm standard deviation (SD) is displayed. Statistical analysis of PLA and immunofluorescence experiments were performed by using two-tailed unpaired t-tests with Welch's correction when variances were significantly different. 1 way or 2-way ANOVA with Dunnett's multiple comparisons test were used for IncuCyte ${ }^{\circledR}$ or luciferase assays respectively $(\alpha=0.05)$. Statistics for change in protein abundance were based on two-tailed t-tests with p-values adjusted with a BenjaminiHochberg FDR.

\section{DATA AVAILABILITY}

The datasets produced in this study are available in the PRIDE database ${ }^{43}$ as detailed : (PXD020155, username: reviewer98271@ebi.ac.uk, password:wDt8mtLF).

\section{ACKNOWLEDGMENTS}

This work was funded by grants from the Ligue Nationale Contre le Cancer (Equipe labellisée), Fondation ARC, Gefluc and the Société Française de Dermatologie. C.E. and L.M. were supported by a fellowship from the Ministère Français de l'Enseignement Supérieur, de la Recherche et de l'Innovation, and Fondation ARC. This work was also supported by "Région Ile-de-France" and Fondation pour la Recherche Médicale grants (to D.L.).

\section{AUTHOR CONTRIBUTIONS}

C.E., A.E. and S.D. conceived the study and designed the experiments. C.E., L.M. and S.D. performed and analyzed the experiments. C.B. provided MITF plasmids and critically revised the manuscript. F.D. carried out the MS experimental work and D.L. supervised MS and data analysis. L.B. and C.M. wrote 
software for immunofluorescence quantification. C.E., A.E. and S.D. wrote the manuscript. C.E., A.E. and S.D. supervised the research.

\section{Conflict of interest}

The authors declare no competing interests.

\section{REFERENCES}

1. Miller, A. J. \& Mihm, M. C. Melanoma. N. Engl. J. Med. 355, 51-65 (2006).

2. Fedorenko, I. V., Gibney, G. T. \& Smalley, K. S. M. NRAS mutant melanoma: biological behavior and future strategies for therapeutic management. Oncogene 32, 3009-3018 (2013).

3. Garnett, M. J. \& Marais, R. Guilty as charged: B-RAF is a human oncogene. Cancer Cell 6, 313319 (2004).

4. Buday, L. \& Downward, J. Many faces of Ras activation. Biochim. Biophys. Acta 1786, 178-187 (2008).

5. Peyssonnaux, C. \& Eychène, A. The Raf/MEK/ERK pathway: new concepts of activation. Biol. Cell 93, 53-62 (2001).

6. Guo, Y.-J. et al. ERK/MAPK signalling pathway and tumorigenesis. Exp. Ther. Med. 19, 1997$2007(2020)$.

7. Dorard, C. et al. RAF proteins exert both specific and compensatory functions during tumour progression of NRAS-driven melanoma. Nat. Commun. 8, 15262 (2017).

8. Marais, R., Light, Y., Paterson, H. F., Mason, C. S. \& Marshall, C. J. Differential regulation of Raf-1, A-Raf, and B-Raf by oncogenic ras and tyrosine kinases. J. Biol. Chem. 272, 4378-4383 (1997).

9. Cheli, Y., Ohanna, M., Ballotti, R. \& Bertolotto, C. Fifteen-year quest for microphthalmiaassociated transcription factor target genes. Pigment Cell Melanoma Res. 23, 27-40 (2010).

10. Goding, C. R. \& Arnheiter, H. MITF-the first 25 years. Genes Dev. 33, 983-1007 (2019). 
11. Hemesath, T. J. et al. microphthalmia, a critical factor in melanocyte development, defines a discrete transcription factor family. Genes Dev. 8, 2770-2780 (1994).

12. King, R., Googe, P. B., Weilbaecher, K. N., Mihm, M. C. \& Fisher, D. E. Microphthalmia transcription factor expression in cutaneous benign, malignant melanocytic, and nonmelanocytic tumors. Am. J. Surg. Pathol. 25, 51-57 (2001).

13. Wellbrock, C. \& Arozarena, I. Microphthalmia-associated transcription factor in melanoma development and MAP-kinase pathway targeted therapy. Pigment Cell Melanoma Res. 28, 390406 (2015).

14. Carreira, S. et al. Mitf regulation of Dial controls melanoma proliferation and invasiveness. Genes Dev. 20, 3426-3439 (2006).

15. Hoek, K. S. et al. Metastatic potential of melanomas defined by specific gene expression profiles with no BRAF signature. Pigment Cell Res. 19, 290-302 (2006).

16. Hoek, K. S. et al. In vivo switching of human melanoma cells between proliferative and invasive states. Cancer Res. 68, 650-656 (2008).

17. Tsoi, J. et al. Multi-stage Differentiation Defines Melanoma Subtypes with Differential Vulnerability to Drug-Induced Iron-Dependent Oxidative Stress. Cancer Cell 33, 890-904.e5 (2018).

18. Rambow, F. et al. Toward Minimal Residual Disease-Directed Therapy in Melanoma. Cell 174, 843-855.e19 (2018).

19. Rambow, F., Marine, J.-C. \& Goding, C. R. Melanoma plasticity and phenotypic diversity: therapeutic barriers and opportunities. Genes Dev. 33, 1295-1318 (2019).

20. Wu, M. et al. c-Kit triggers dual phosphorylations, which couple activation and degradation of the essential melanocyte factor Mi. Genes Dev. 14, 301-312 (2000).

21. Hemesath, T. J., Price, E. R., Takemoto, C., Badalian, T. \& Fisher, D. E. MAP kinase links the transcription factor Microphthalmia to c-Kit signalling in melanocytes. Nature 391, 298-301 (1998).

22. $\mathrm{Xu}, \mathrm{W}$. et al. Regulation of microphthalmia-associated transcription factor MITF protein levels by association with the ubiquitin-conjugating enzyme hUBC9. Exp. Cell Res. 255, 135-143 (2000). 
23. Price, E. R. et al. Lineage-specific signaling in melanocytes. C-kit stimulation recruits p300/CBP to microphthalmia. J. Biol. Chem. 273, 17983-17986 (1998).

24. Ngeow, K. C. et al. BRAF/MAPK and GSK3 signaling converges to control MITF nuclear export. Proc. Natl. Acad. Sci. U. S. A. 115, E8668-E8677 (2018).

25. An, S. et al. A-Raf: A new star of the family of raf kinases. Crit. Rev. Biochem. Mol. Biol. 50, $520-531(2015)$.

26. Zhang, X.-Y. et al. Revealing A-Raf functions through its interactome. Biochim. Biophys. Acta Proteins Proteomics 1866, 849-856 (2018).

27. Zhang, X.-Y. et al. Comparative interactome analysis reveals distinct and overlapping properties of Raf family kinases. Biochem. Biophys. Res. Commun. 514, 1217-1223 (2019).

28. Laurette, P. et al. Transcription factor MITF and remodeller BRG1 define chromatin organisation at regulatory elements in melanoma cells. eLife 4, (2015).

29. Davies, H. et al. Mutations of the BRAF gene in human cancer. Nature 417, 949-954 (2002).

30. Hmitou, I., Druillennec, S., Valluet, A., Peyssonnaux, C. \& Eychène, A. Differential regulation of B-raf isoforms by phosphorylation and autoinhibitory mechanisms. Mol. Cell. Biol. 27, 31-43 (2007).

31. Garraway, L. A. et al. Integrative genomic analyses identify MITF as a lineage survival oncogene amplified in malignant melanoma. Nature 436, 117-122 (2005).

32. Carreira, S. et al. Mitf cooperates with Rb1 and activates p21Cip1 expression to regulate cell cycle progression. Nature 433, 764-769 (2005).

33. Cheli, Y. et al. Mitf is the key molecular switch between mouse or human melanoma initiating cells and their differentiated progeny. Oncogene 30, 2307-2318 (2011).

34. Cheli, Y. et al. Hypoxia and MITF control metastatic behaviour in mouse and human melanoma cells. Oncogene 31, 2461-2470 (2012).

35. Haq, R. et al. Oncogenic BRAF regulates oxidative metabolism via PGC1 $\alpha$ and MITF. Cancer Cell 23, 302-315 (2013).

36. McGill, G. G. et al. Bcl2 regulation by the melanocyte master regulator Mitf modulates lineage survival and melanoma cell viability. Cell 109, 707-718 (2002). 
37. Möller, K. et al. MITF has a central role in regulating starvation-induced autophagy in melanoma. Sci. Rep. 9, 1055 (2019).

38. Strub, T. et al. Essential role of microphthalmia transcription factor for DNA replication, mitosis and genomic stability in melanoma. Oncogene 30, 2319-2332 (2011).

39. Giuliano, S. et al. Microphthalmia-associated transcription factor controls the DNA damage response and a lineage-specific senescence program in melanomas. Cancer Res. 70, 3813-3822 (2010).

40. Poullet, P., Carpentier, S. \& Barillot, E. myProMS, a web server for management and validation of mass spectrometry-based proteomic data. Proteomics 7, 2553-2556 (2007).

41. Spivak, M., Weston, J., Bottou, L., Käll, L. \& Noble, W. S. Improvements to the percolator algorithm for Peptide identification from shotgun proteomics data sets. J. Proteome Res. 8, 37373745 (2009).

42. Valot, B., Langella, O., Nano, E. \& Zivy, M. MassChroQ: a versatile tool for mass spectrometry quantification. Proteomics 11, 3572-3577 (2011).

43. Perez-Riverol, Y. et al. The PRIDE database and related tools and resources in 2019: improving support for quantification data. Nucleic Acids Res. 47, D442-D450 (2019). 


\section{FIGURE LEGENDS}

\section{Fig.1 Identification of MITF as an ARAF partner.}

a Schematic representation of the workflow to identify new ARAF relevant partners. Endogenous ARAF was immunoprecipitated from ARAF-only or control cells lysates. Binding partners were obtained by using quantitative label-free mass spectrometry analysis performed from five ARAF-only and four control cells replicates. Volcano plot analysis represents 2700 quantified proteins in control and ARAFonly cells with $\mathrm{X}$ axis indicating the $\log 2$ fold change (FC) (ARAF-only versus control cells) and $\mathrm{Y}$ axis the $-\log 10$ of adjusted $p$-value. The non-axial vertical lines (in green) denote absolute fold change of 2 while the non-axial horizontal line (in pink) denotes the adjusted p-value of ratio significance of 0.001 . External plots show unique proteins with peptides identified only in one sample type (left in control and right in ARAF-only cells). 99 proteins were selected for further analysis through a siRNA based screen. The 69 proteins enriched in ARAF-only cells where labelled in brown, unique in ARAF-only cells in pink and ARAF interactors published by Zhang et al. ${ }^{26}$ in green. ARAF-only cells proliferation was measured during 72 hours by using IncuCyte ${ }^{\circledR}$ technology after transfection with siRNA pools targeting each of the 99 putative partners (Supplementary Fig. 1). The theorical curve shows the percentage of occupied surface over time for a given knockdown target. SiRNA having a pro-proliferative (si up) or anti-proliferative (si down) effect compared to a negative control (si CTL, in black) are highlighted in green and red, respectively. b List of the 99 putative ARAF partners selected for the siRNA-based functional screen. 69 were enriched in ARAF-only cells with the following parameters number of peptides $>9$, ratio $>2$ and adjusted p-value $<0.001,15$ exclusively identified in ARAF-only cells, and 15 are commonly found with the ARAF interactome published by Zhang et al. ${ }^{26}$. Proteins inducing an antior pro-proliferative effect after silencing by siRNA are highlighted in green and red, respectively. c Proliferation of ARAF-only cells after transfection with a control siRNA (siCTL, in black), individual siRNA against MITF (siMITF \#1 or siMITF \#2, in red) or siRNA pool against ARAF (siARAF, in blue).

Data are the mean $\pm \mathrm{SD}$ of four replicates $(n=4) .{ }^{* * * *}$ p-value $<0.0001$ compared by a 2 -way ANOVA with Dunnett's multiple comparisons test. d Western blot analysis of MITF and ARAF protein levels in ARAF-only cells transfected with either siCTL, siMITF or siARAF. $\beta$-actin is used as a loading control. 


\section{Fig.2 Validation of the ARAF/MITF interaction.}

a Identification of endogenous ARAF/MITF complexes by co-immunoprecipitation. ARAF-only cells extracts were immunoprecipitated with an anti-ARAF antibody. Immune complexes (IP) and total extracts (TE) were immunoblotted with anti-MITF and anti-ARAF antibodies. b Identification of endogenous ARAF/MITF complexes by Proximity Ligation Assay. ARAF/MITF complexes were visualized as red dots in ARAF-only cells compared to control cells expressing an shRNA against ARAF by using a fluorescent microscope. Cell nuclei were stained with DAPI. Scatter plots represent the average number of dots per nucleus of six replicates $(n=6)$. Means with standard deviations are shown. *p-value $=0.002$ compared by unpaired t-test with Welch's correction. c Identification of a direct ARAF/MITF interaction by in vitro coimmunoprecipitation. Recombinant MITF and flag-tagged ARAF proteins were incubated in NP40 Buffer. ARAF was immunoprecipitated with an anti-flag antibody. Immune complexes and recombinant proteins were blotted with anti-MITF and anti-ARAF antibodies.

\section{Fig.3 Characterization of RAF/MITF interaction.}

a, b, c MITF interaction with ARAF, BRAF or CRAF. HEK293T cells were cotransfected with the mycMITF construct and each of the three HA-ARAF, HA-BRAF or HA-CRAF constructs (panels A, B, C respectively). RAF proteins were immunoprecipitated with anti-HA antibody. Immune complexes and total extracts were immunoblotted with anti-myc or anti-HA. Quantifications of the protein ratio in the immunoprecipitation over the total extract IP/TE confirmed the specificity of these interactions. d HEK293T cells are cotransfected with the HA-MITF construct and each of the three myc-BRAF ${ }^{\mathrm{WT}}$, myc-BRAF ${ }^{\mathrm{V} 600 \mathrm{E}}$ or myc-BRAF ${ }^{\mathrm{KD}}$ constructs. Cell lysates were immunoprecipitated with anti-myc antibody. Immune complexes and total extracts were revealed with anti-HA and anti-myc antibodies. Coimmunoprecipitations were quantified using Image $\mathrm{J}$ software by measuring signal intensity in immunoprecipitation over total extract and normalized on control lane. e HEK293T cells were cotransfected with the myc-MITF construct and HA-Nter or flag-Cter or both constructs. Cell lysates were immunoprecipitated either with anti-flag or anti-HA antibodies, and immune complexes were 
revealed with anti-MITF, anti-HA or anti-flag antibodies. Transfection efficiency was monitored by direct western blotting of total protein extracts.

\section{Fig.4 Effect of RAF proteins on MITF subcellular localization.}

Subcellular localization of MITF in the presence of ARAF (a), BRAF (b) or CRAF (c). HEK293T cells were cotransfected with myc-MITF and HA-ARAF, HA-BRAF or HA-CRAF. Cell immunostaining was performed with anti-myc or anti-MITF and anti-ARAF, anti-BRAF or anti-CRAF. Scatter plots represent the ratio $\mathrm{MITF}_{\text {nud }} / \mathrm{MITF}_{\text {cyt }}$ calculated by measuring the quantity of nuclear MITF over cytoplasmic MITF. Means with standard deviations are shown. Scale bar: $200 \mu \mathrm{m}$; **** pvalue $<0.0001, * *$ p-value $=0.014$ or 0.029 (for BRAF and CRAF, respectively) compared by unpaired ttest with Welch's correction.

Fig. 5 Effect of RAF proteins on MITF transcriptional activity. HEK293T cells were cotransfected with $5 \mathrm{ng}$ of MITF plasmid and increasing doses of either ARAF, CRAF, BRAF ${ }^{\mathrm{WT}}, \mathrm{BRAF}^{\mathrm{V} 600 \mathrm{E}}$ or BRAF $^{\mathrm{KD}}$ constructs in the presence of a TYR-Luc luciferase reporter and a control $\beta$-actin- $\beta$ galactosidase reporter. The ratio of luciferase to $\beta$-galactosidase activities is shown as the mean $\pm \mathrm{SD}$ of three replicates $(n=3)$. One-way ANOVA test was performed to compare all conditions and Dunnett's tests for the multiple comparisons to the no MITF condition (n.s., non-significant; * p-value $<0.05$; **

p-value $<0.01 ; * * *$ p-value $<0.001 ; * * * *$ p-value $<0.0001)$.

\section{Table 1. List of the 2700 proteins identified by mass spectrometry.}




\section{SUPPLEMENTARY INFORMATION}

\section{SUPPLEMENTARY FIGURES LEGENDS}

\section{Supplementary Fig. 1 Functional siRNA-based screen for a subset of 16 interactors identified by proteomics.}

Proliferation followed by IncuCyte ${ }^{\circledR}$ of ARAF-only cells after transfection with a pool of siRNA against a selected interactor (red or green), control siRNA (siCTL, black) or siRNA pool against ARAF (siARAF, blue). Knock-down of the selected interactors induced either anti-proliferative (for 5 interactors highlighted in red, top panel) or pro-proliferative effect (for 11 interactors highlighted in green, bottom panel). Data are the mean $\pm \mathrm{SD}$ of three or four replicates. ${ }^{*}$ p-value $<0.05, * * \mathrm{p}$ value $<0.01, * * *$ p-value $<0.001, * * * *$ p-value $<0.0001$ compared by a 2 -way ANOVA with Dunnett's multiple comparisons test.

\section{Supplementary Fig. 2 Identification of endogenous BRAF/MITF and CRAF/MITF complexes by Proximity Ligation Assay.}

Complexes were visualized as red dots by using a fluorescent microscope in wild type cells, namely a NRAS-mutated murine cell line that expresses ARAF, BRAF and CRAF. Cell nuclei were stained with DAPI. ARAF-only cells in which BRAF and CRAF have been deleted were used as a control. Scatter plots represent the average number of dots per nucleus of at least five replicates $(n=5)$. Means with standard deviations are shown. $* * * \mathrm{p}$-value $=0.0002, * * \mathrm{p}$-value $<0.0014$ compared by unpaired t-test. 
a

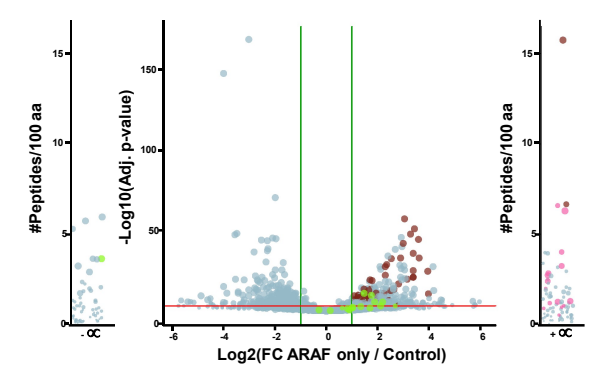

2601 quantified proteins

69 ARAF only partners

15 unique ARAF partners

15 ARAF partners from Zhang et al.
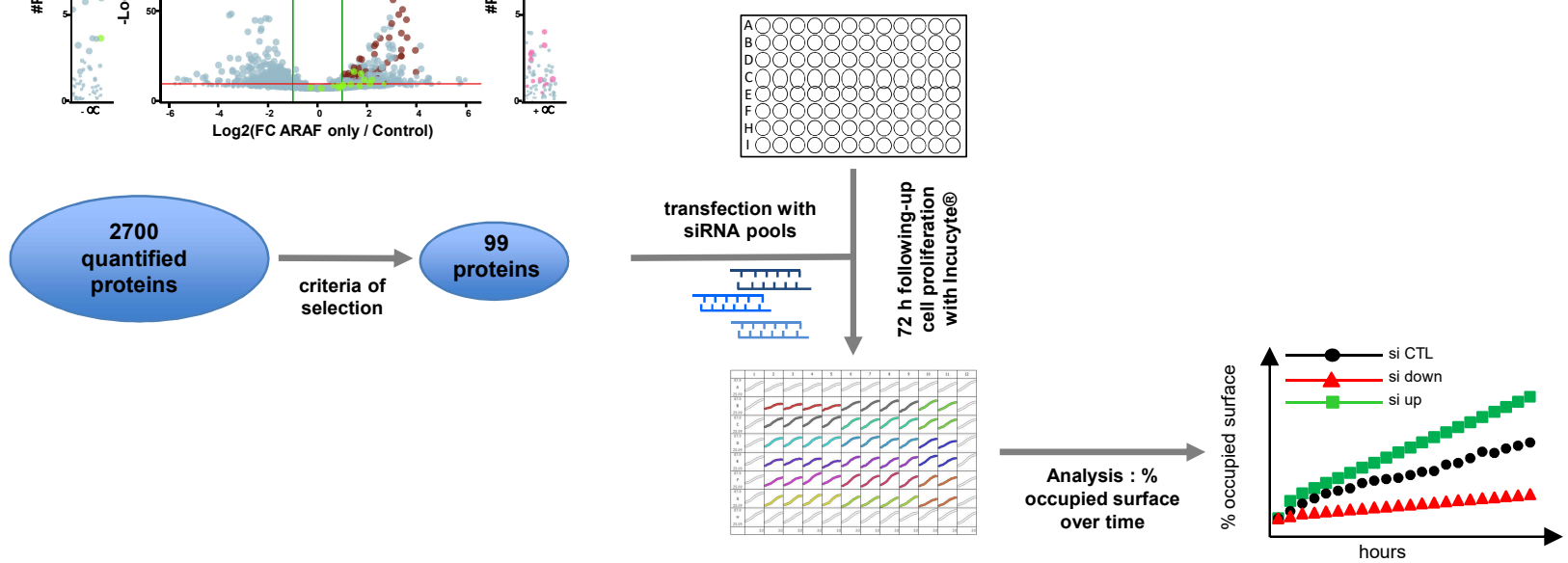

b

69 ARAF partners enriched in ARAF only cells

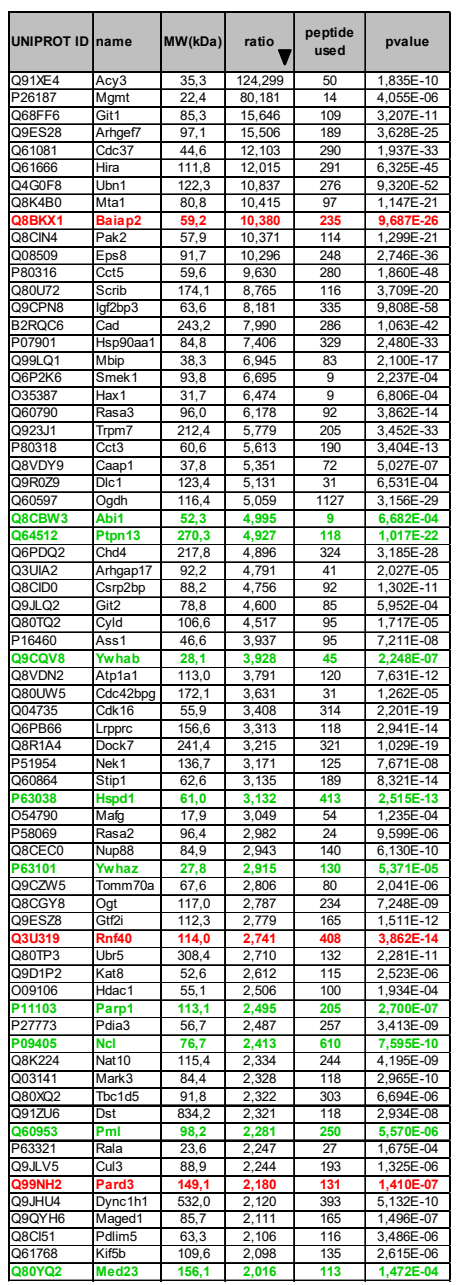

C

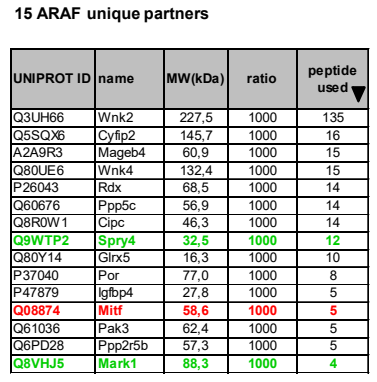

15 ARAF partners from Zhang et al.

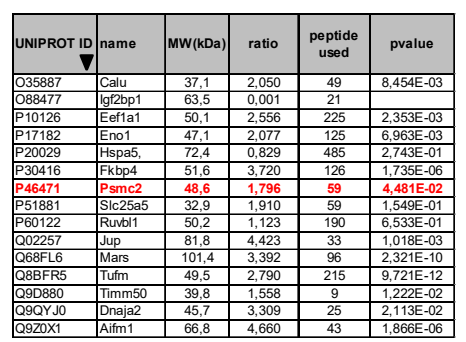

d

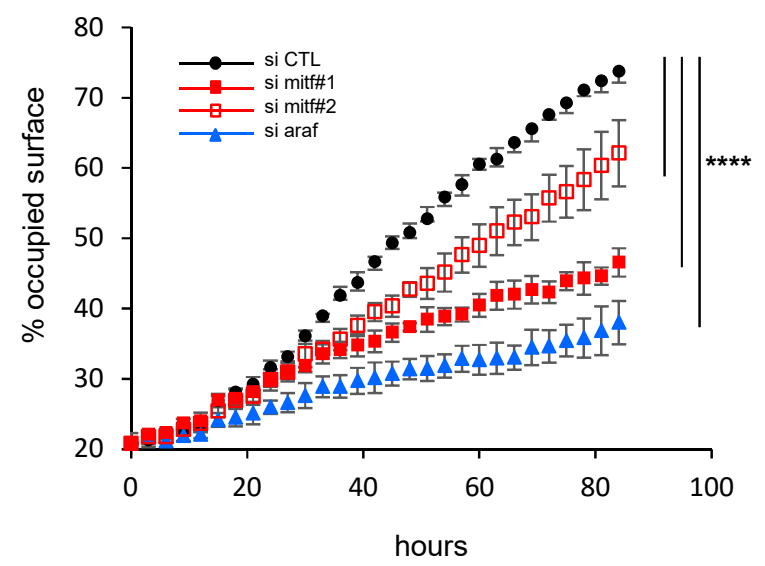

si $\quad$ - ctl mitf\#1 mitf\#2 araf

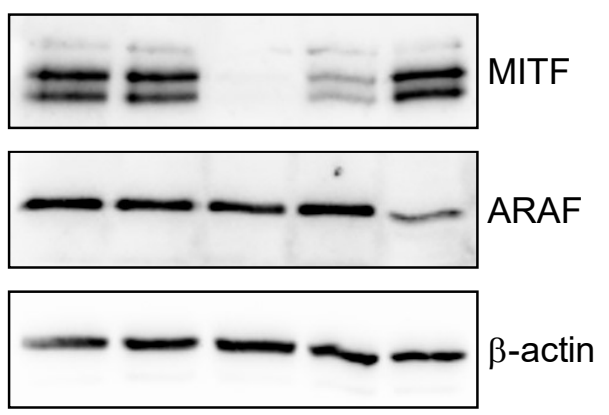

Figure 1 Estrada et al. 
a

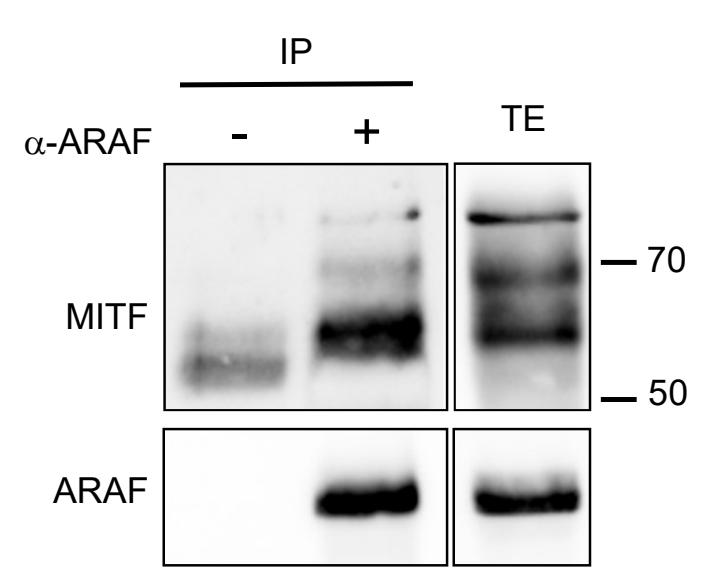

b

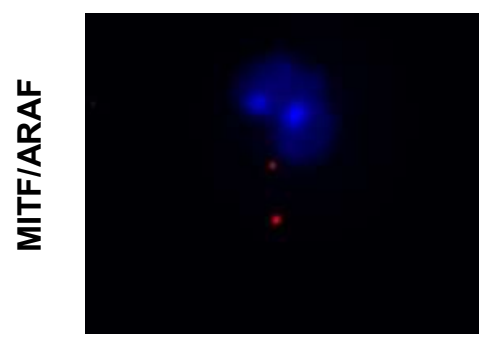

Control
C
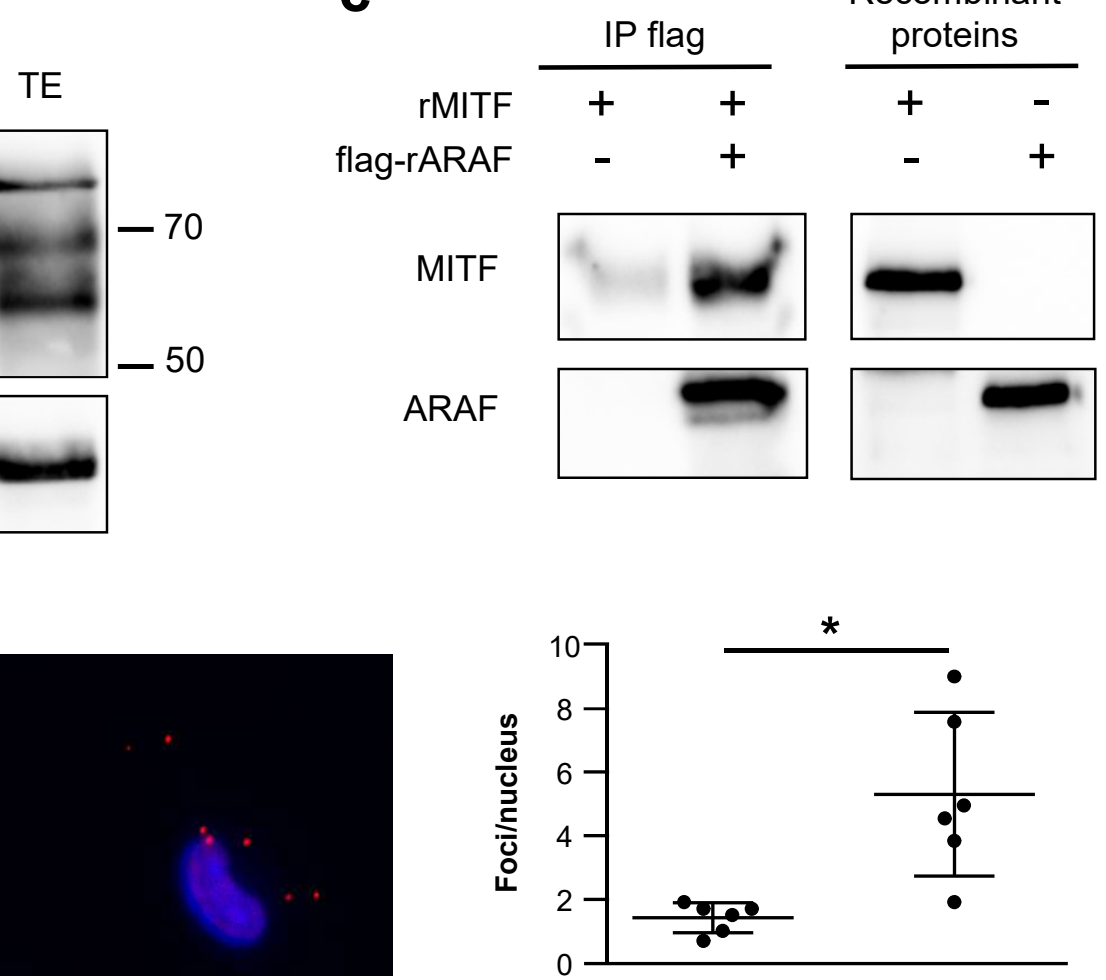

ARAF only

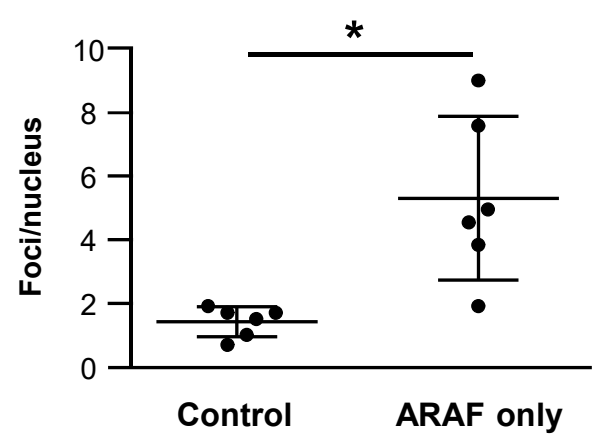

Figure 2 Estrada et al. 
a

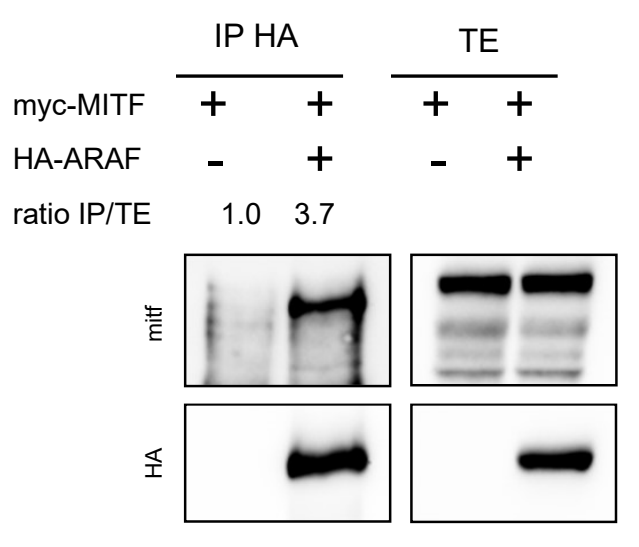

b

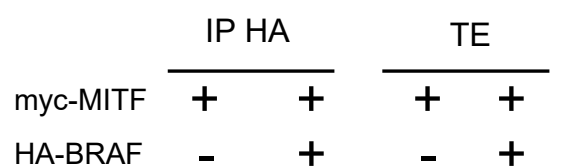

C

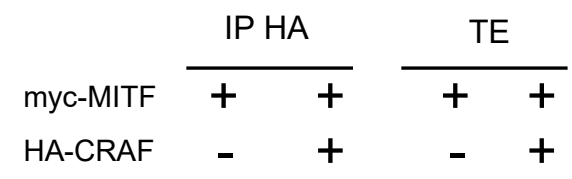

$1.0 \quad 2.0$
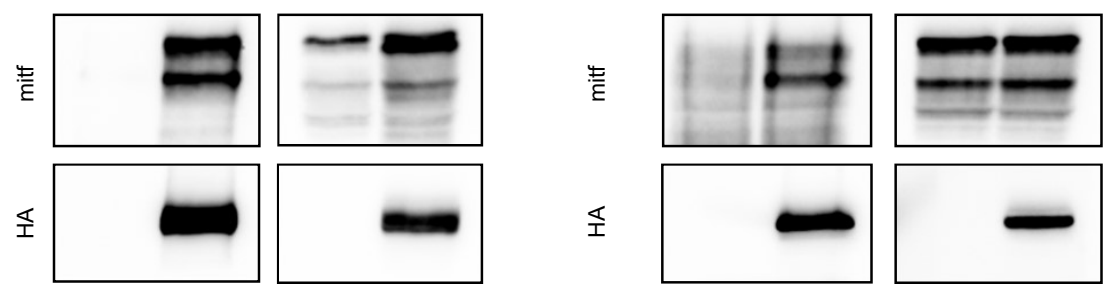

d

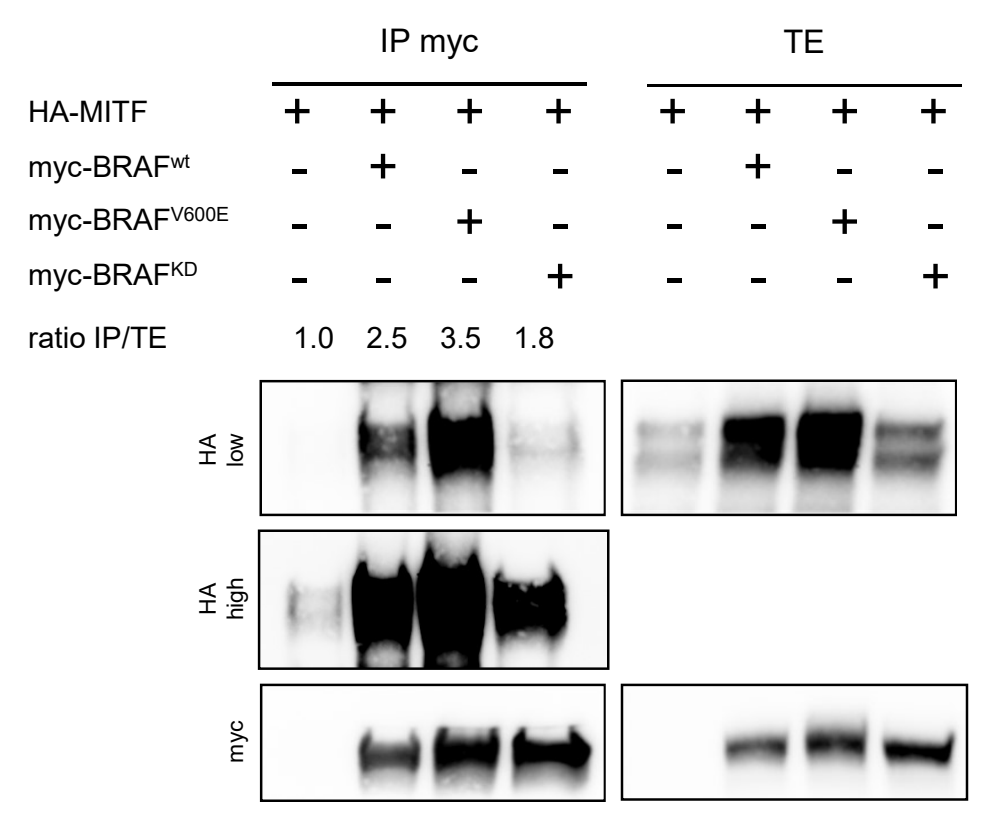

e

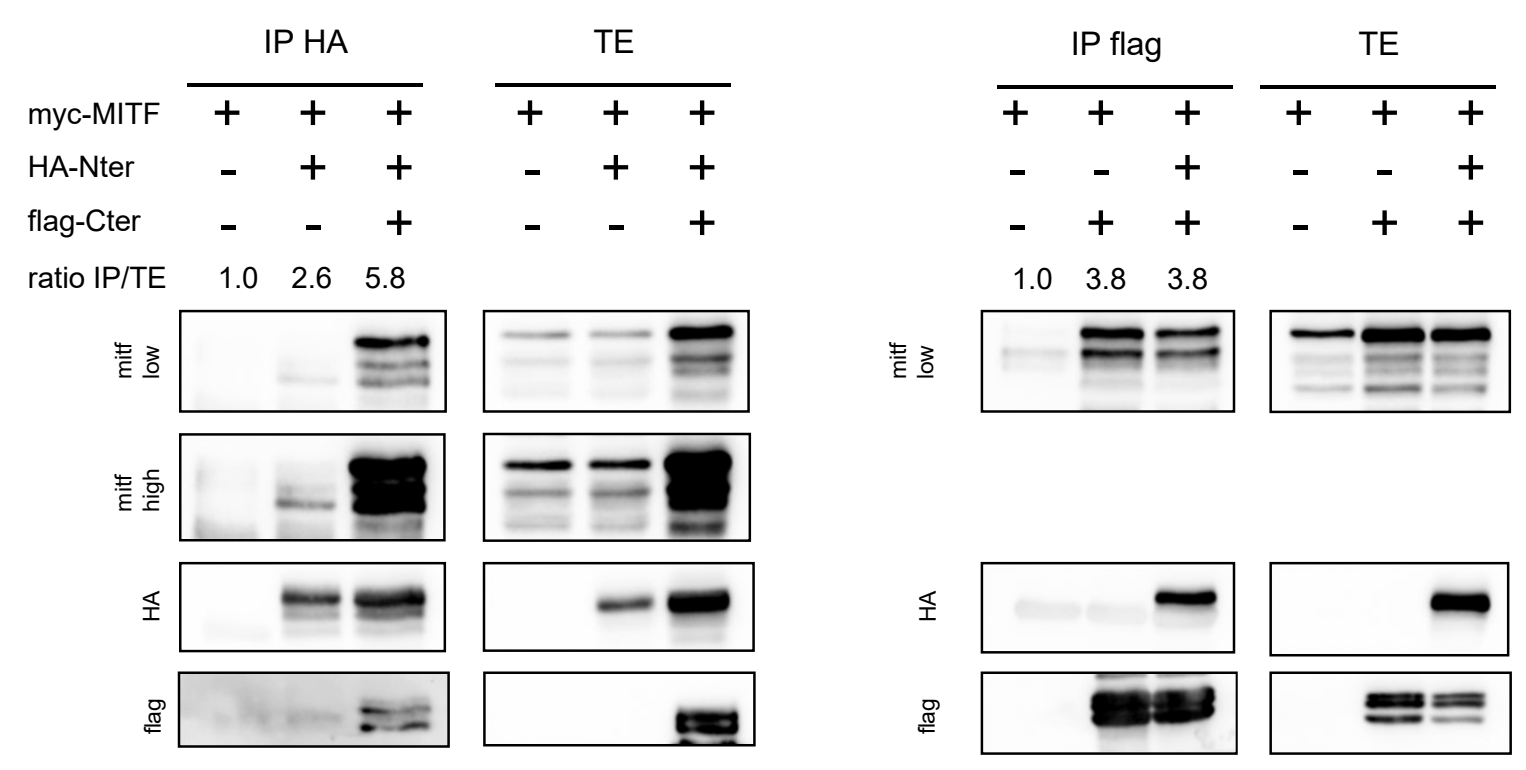

Figure 3 Estrada et al. 
a

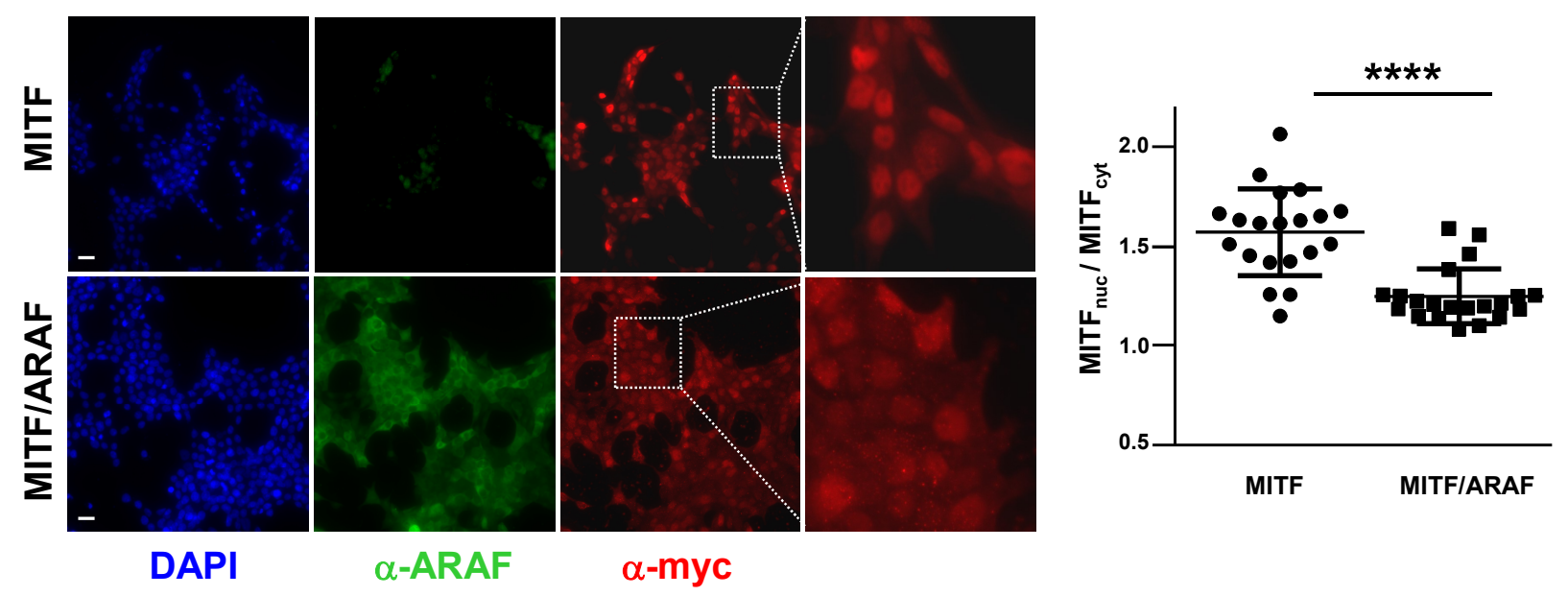

b
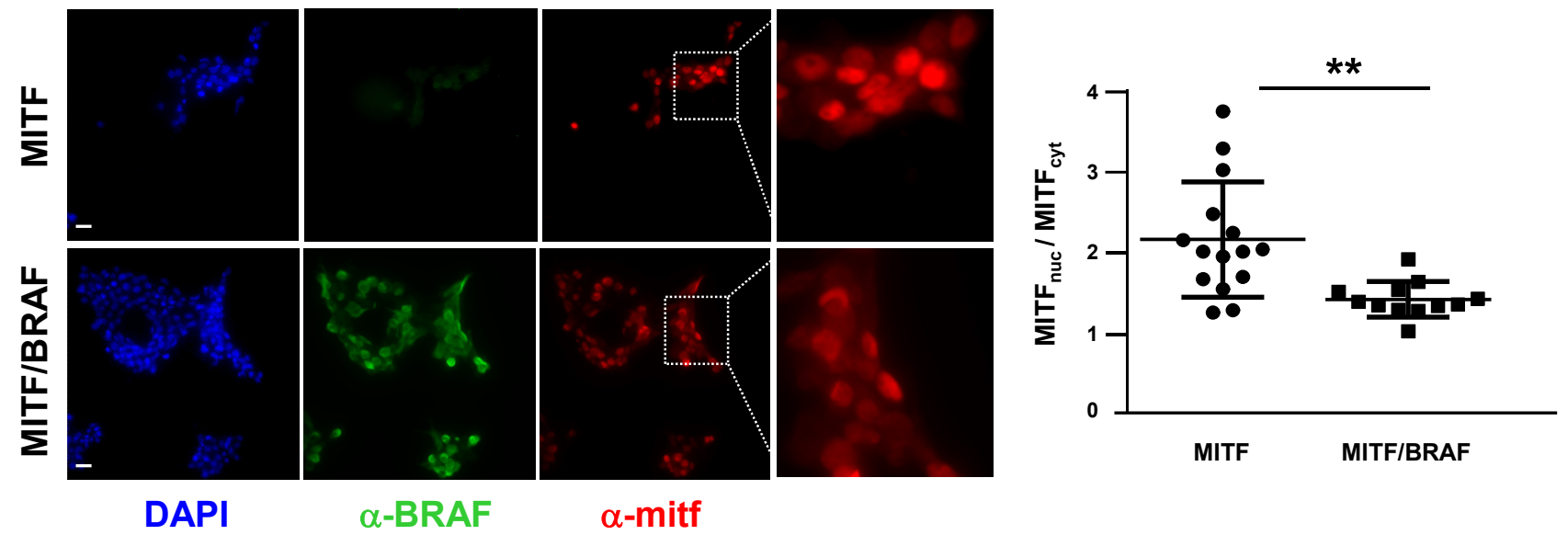

C
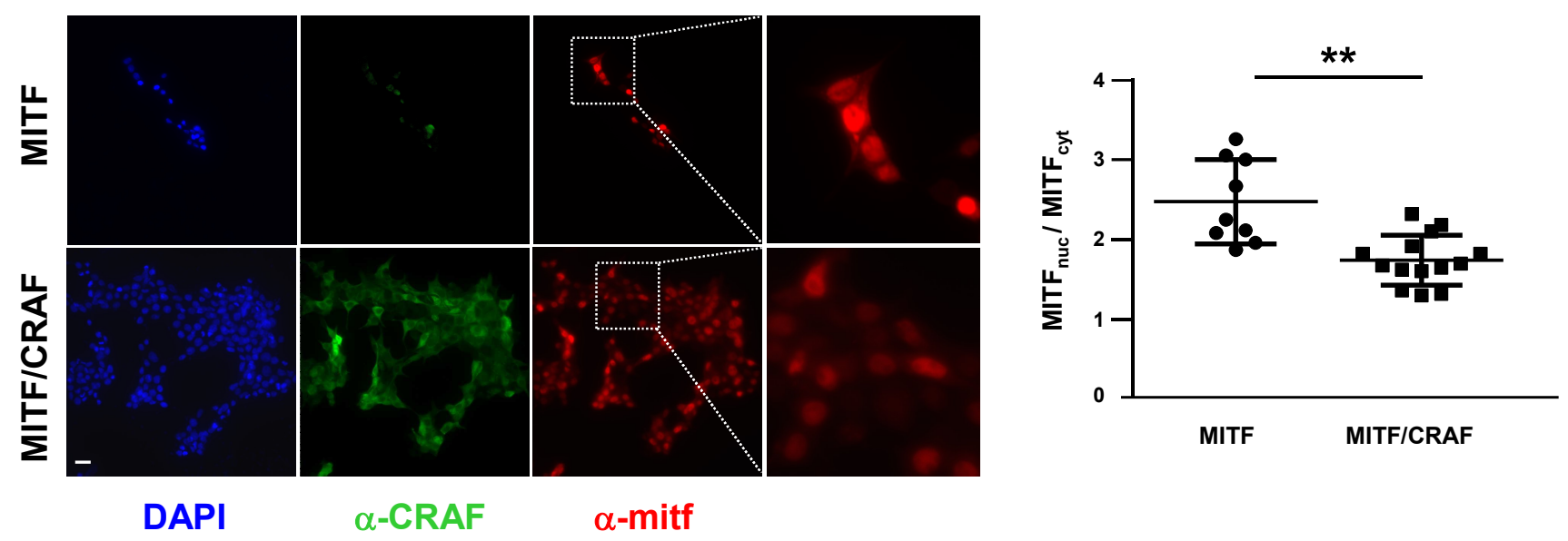

Figure 4 Estrada et al. 

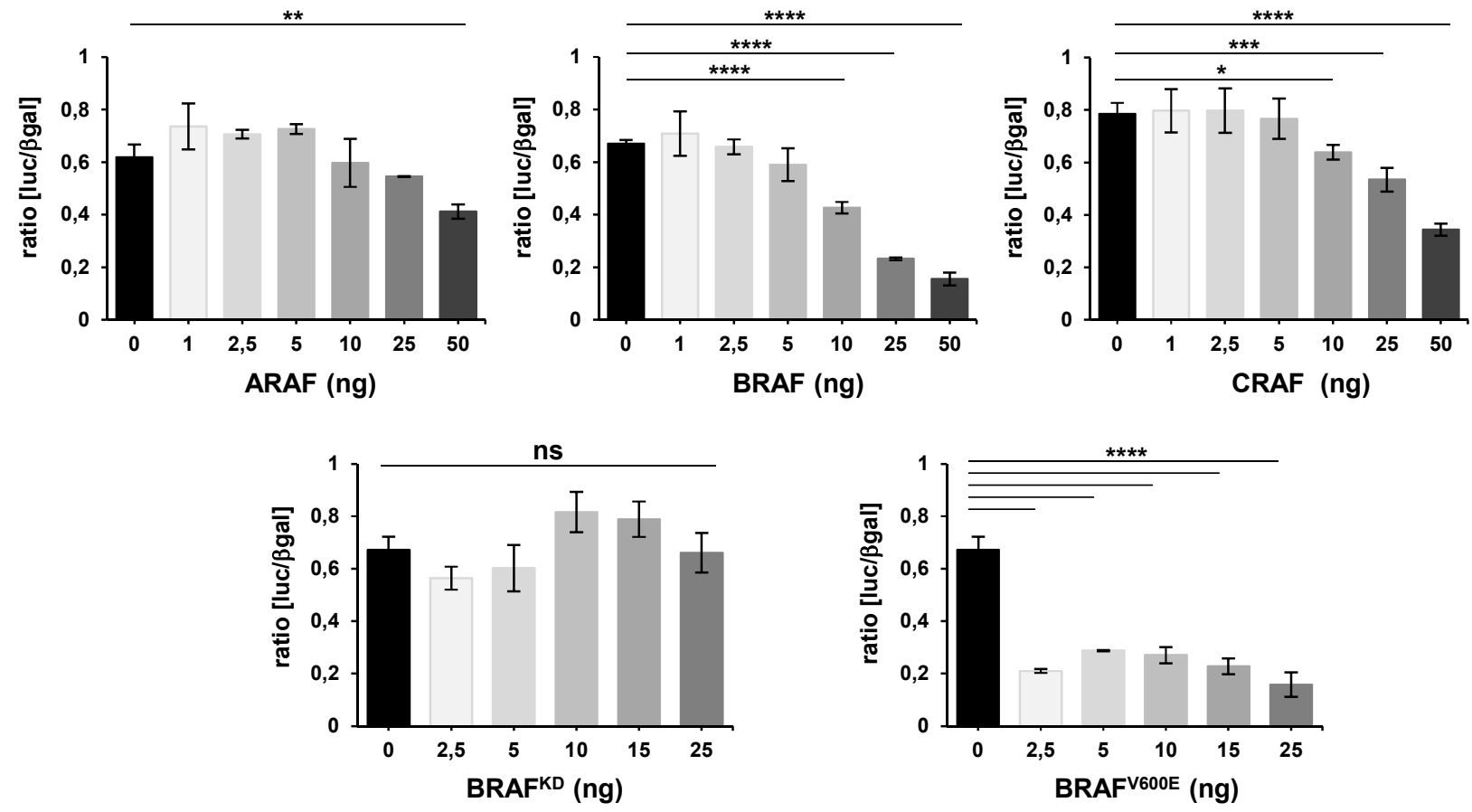

Figure 5 Estrada et al. 

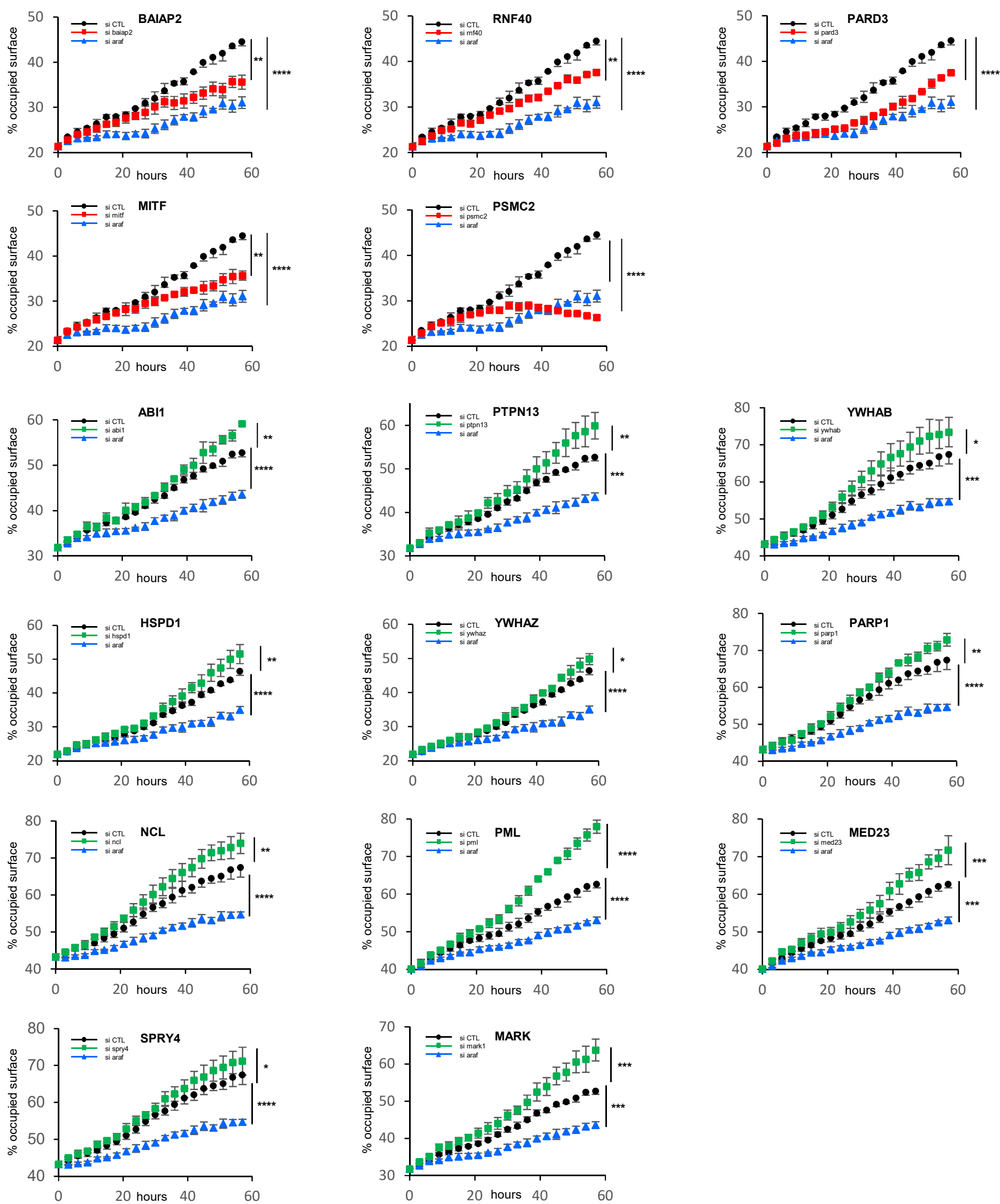

\section{Supplementary Fig. 1 Functional siRNA-based screen for a subset of 16 interactors identified by proteomics.}

Proliferation followed by IncuCyte ${ }^{\circledR}$ of ARAF-only cells after transfection with a pool of siRNA against a selected interactor (red or green), control siRNA (siCTL, black) or siRNA pool against ARAF (siARAF, blue). Knock-down of the selected interactors induced either anti-proliferative (for 5 interactors highlighted in red, top panel) or pro-proliferative effect (for 11 interactors highlighted in green, bottom panel). Data are the mean $\pm \mathrm{SD}$ of three or four replicates. * p-value $<0.05, * * \mathrm{p}$ value $<0.01, * * *$ p-value $<0.001, * * * *$ p-value $<0.0001$ compared by a 2-way ANOVA with Dunnett's multiple comparisons test. 


\section{Supplementary Fig. 2 Estrada et al.}

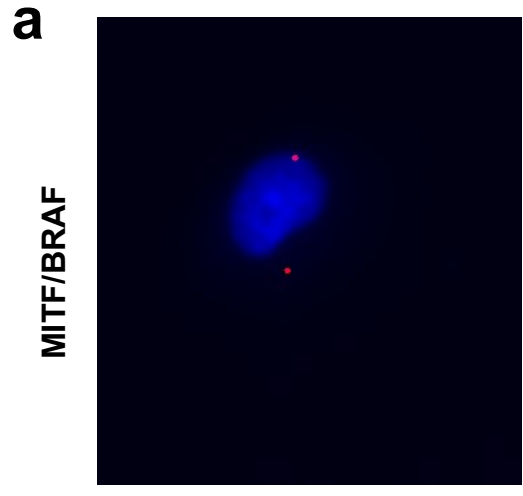

control

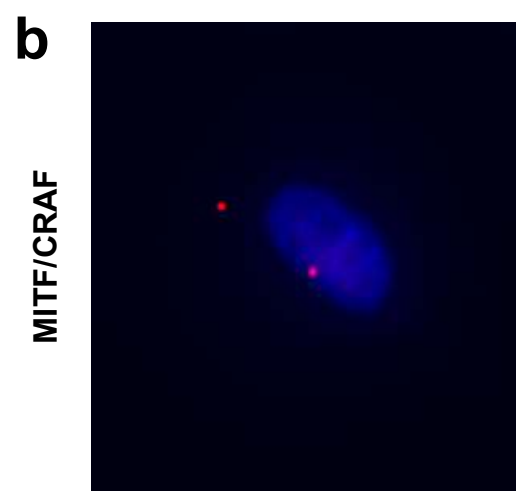

control

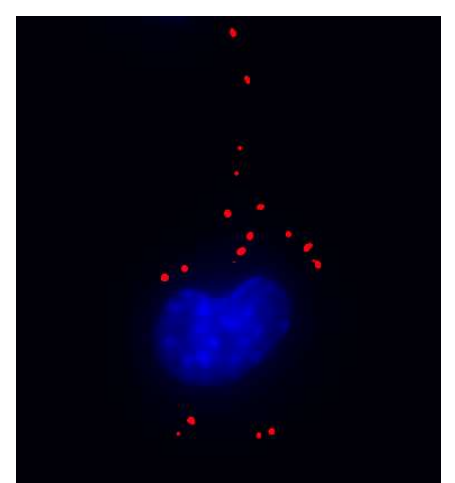

wild type

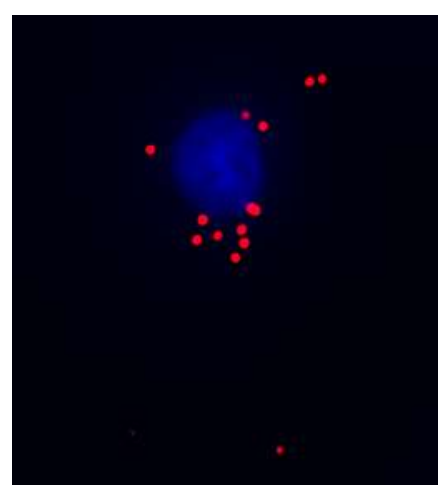

wild type

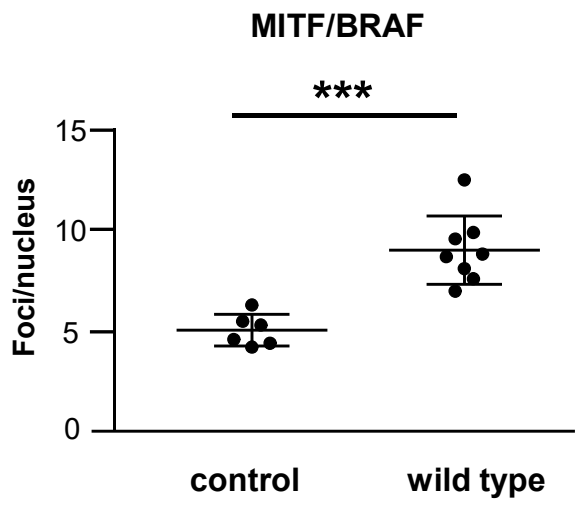

MITF/CRAF

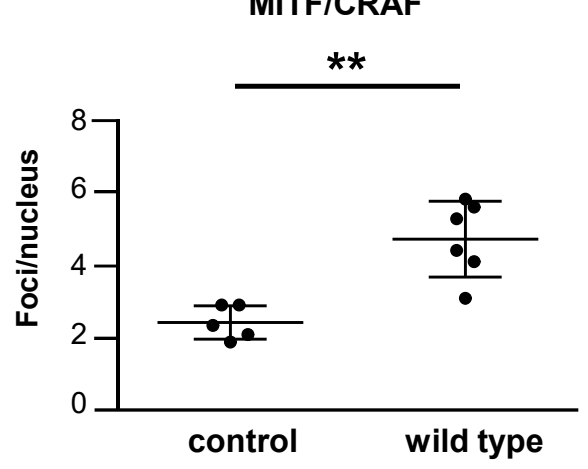

Supplementary Fig. 2 Identification of endogenous BRAF/MITF and CRAF/MITF complexes by Proximity Ligation Assay.

Complexes were visualized as red dots by using a fluorescent microscope in wild type cells, namely a NRAS-mutated murine cell line that expresses ARAF, BRAF and CRAF. Cell nuclei were stained with DAPI. ARAF-only cells in which BRAF and CRAF have been deleted were used as a control. Scatter plots represent the average number of dots per nucleus of at least five replicates $(n=5)$. Means with standard deviations are shown. $* * * \mathrm{p}$-value $=0.0002, * * \mathrm{p}$-value $<0.0014$ compared by unpaired $\mathrm{t}$-test. 


\section{Figures}

a
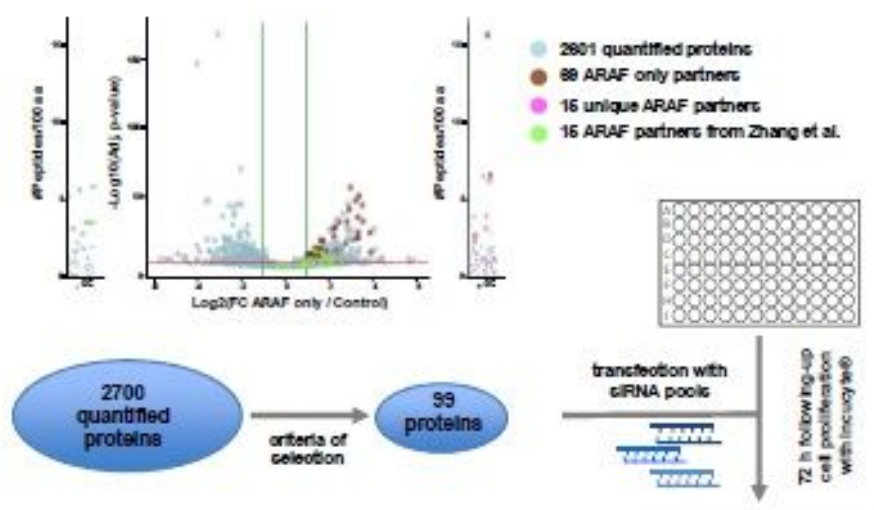

b

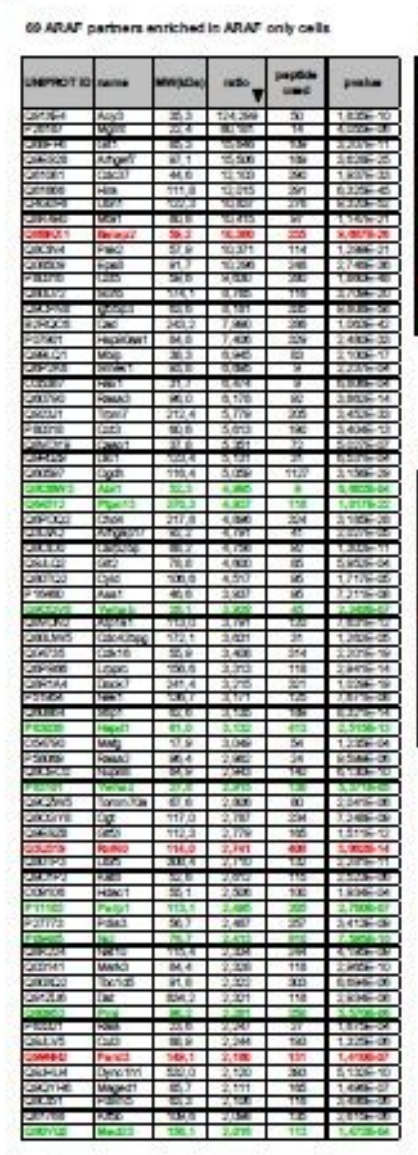

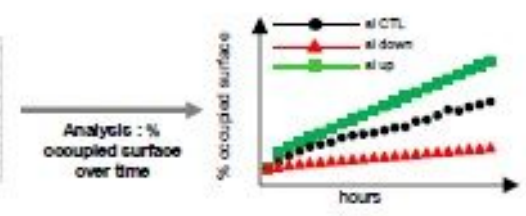

C

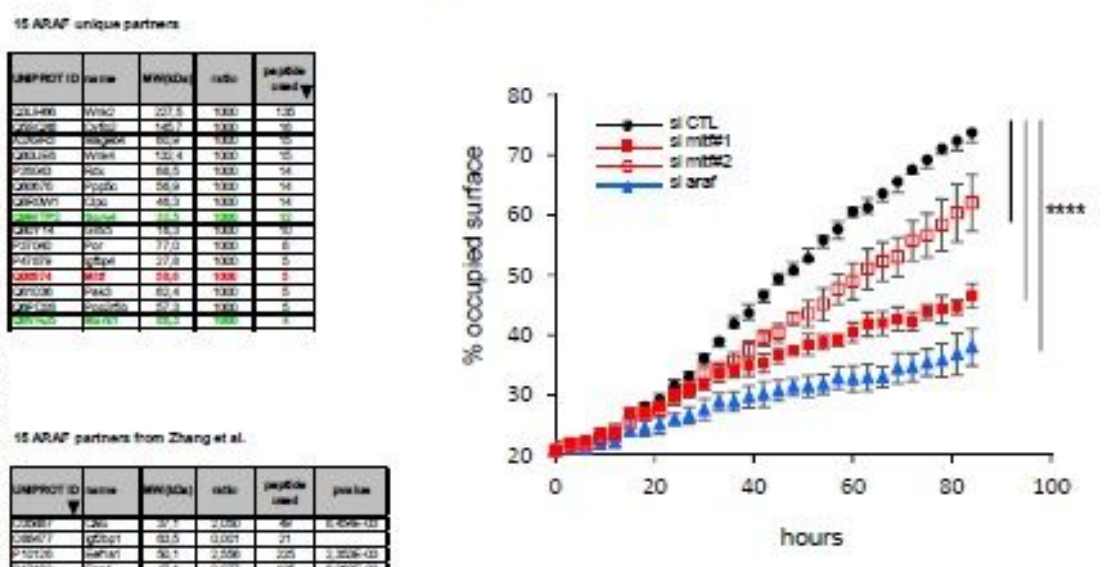

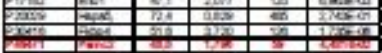
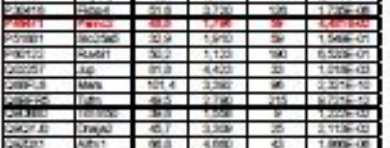

d

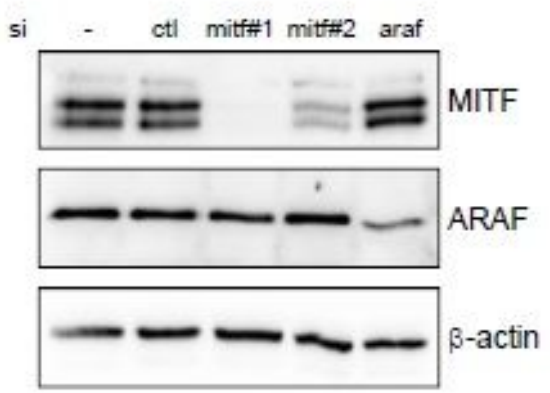

\section{Figure 1}

Identification of MITF as an ARAF partner. a Schematic representation of the workflow to identify new ARAF relevant partners. Endogenous ARAF was immunoprecipitated from ARAF-only or control cells lysates. Binding partners were obtained by using quantitative label-free mass spectrometry analysis 
performed from five ARAF-only and four control cells replicates. Volcano plot analysis represents 2700 quantified proteins in control and ARAFonly cells with $X$ axis indicating the log2 fold change (FC) (ARAFonly versus control cells) and $Y$ axis the - log10 of adjusted $p$-value. The non-axial vertical lines (in green) denote absolute fold change of 2 while the non-axial horizontal line (in pink) denotes the adjusted p-value of ratio significance of 0.001 . External plots show unique proteins with peptides identified only in one sample type (left in control and right in ARAF-only cells). 99 proteins were selected for further analysis through a siRNA based screen. The 69 proteins enriched in ARAF-only cells where labelled in brown, unique in ARAF-only cells in pink and ARAF interactors published by Zhang et al. 26 in green. ARAF-only cells proliferation was measured during 72 hours by using IncuCyte ${ }^{\circledR}$ technology after transfection with siRNA pools targeting each of the 99 putative partners (Supplementary Fig. 1). The theorical curve shows the percentage of occupied surface over time for a given knockdown target. SiRNA having a proproliferative (si up) or anti-proliferative (si down) effect compared to a negative control (si CTL, in black) are highlighted in green and red, respectively. $b$ List of the 99 putative ARAF partners selected for the siRNA-based functional screen. 69 were enriched in ARAF-only cells with the following parameters number of peptides $>9$, ratio $>2$ and adjusted p-value $<0.001,15$ exclusively identified in ARAF-only cells, and 15 are commonly found with the ARAF interactome published by Zhang et al. 26. Proteins inducing an antior pro-proliferative effect after silencing by siRNA are highlighted in green and red, respectively. $C$ Proliferation of ARAF-only cells after transfection with a control siRNA (siCTL, in black), individual siRNA against MITF (siMITF \#1 or siMITF \#2, in red) or siRNA pool against ARAF (siARAF, in blue). Data are the mean \pm SD of four replicates $(n=4)$. $* \star \star \star ~ p$-value $<0.0001$ compared by a 2-way ANOVA with Dunnett's multiple comparisons test. $d$ Western blot analysis of MITF and ARAF protein levels in ARAF-only cells transfected with either siCTL, siMITF or siARAF. $\beta$-actin is used as a loading control. 
a

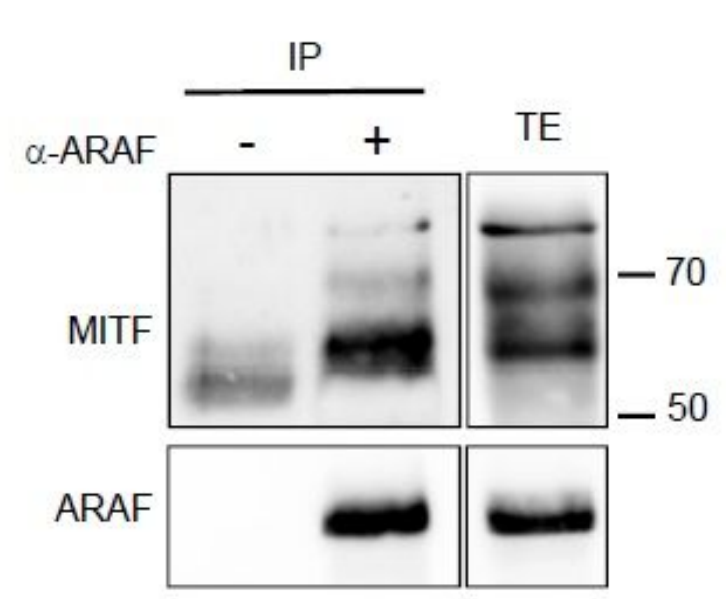

b

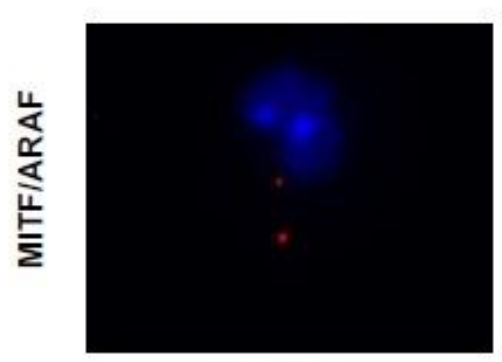

Control

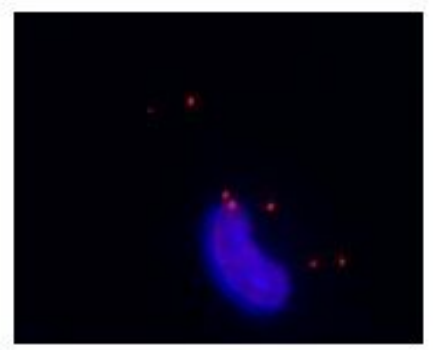

ARAF only
C

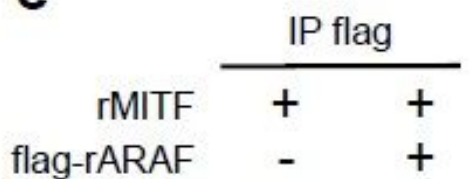

MITF

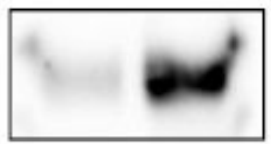

ARAF
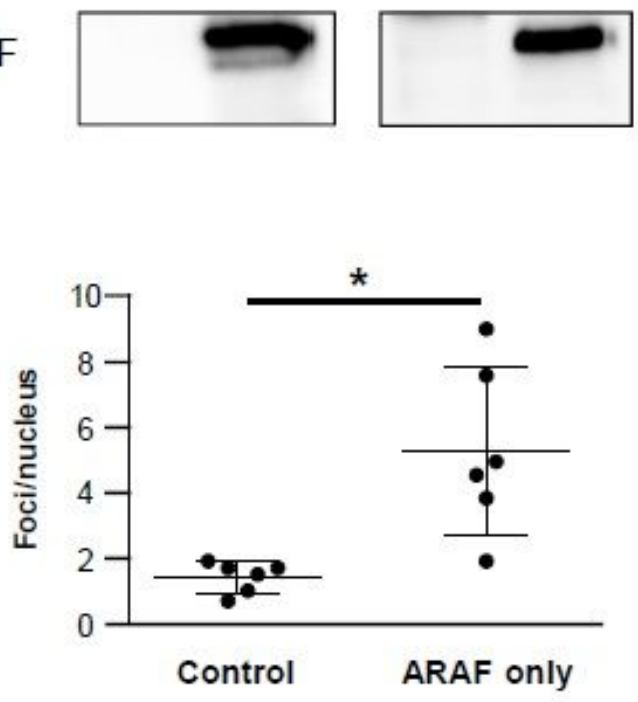

Figure 2

Validation of the ARAF/MITF interaction. a Identification of endogenous ARAF/MITF complexes by coimmunoprecipitation. ARAF-only cells extracts were immunoprecipitated with an anti-ARAF antibody. Immune complexes (IP) and total extracts (TE) were immunoblotted with anti-MITF and anti-ARAF antibodies. b Identification of endogenous ARAF/MITF complexes by Proximity Ligation Assay. ARAF/MITF complexes were visualized as red dots in ARAF-only cells compared to control cells expressing an shRNA against ARAF by using a fluorescent microscope. Cell nuclei were stained with DAPI. Scatter plots represent the average number of dots per nucleus of six replicates $(n=6)$. Means with standard deviations are shown. *p-value $=0.002$ compared by unpaired t-test with Welch's correction. $c$ Identification of a direct ARAF/MITF interaction by in vitro coimmunoprecipitation. Recombinant MITF and flag-tagged ARAF proteins were incubated in NP40 Buffer. ARAF was immunoprecipitated with an anti-flag antibody. Immune complexes and recombinant proteins were blotted with anti-MITF and antiARAF antibodies. 
a

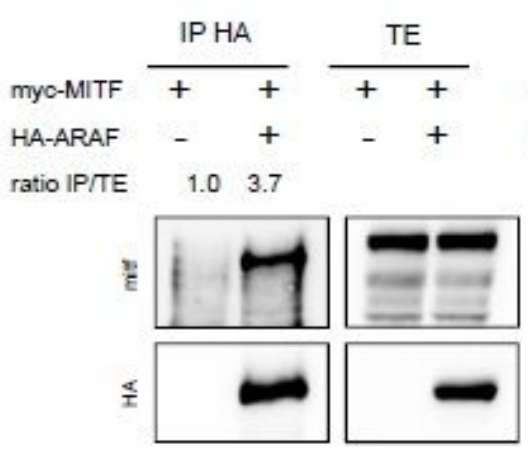

b
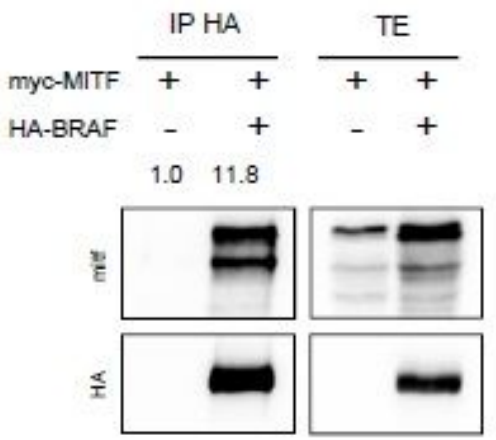

C

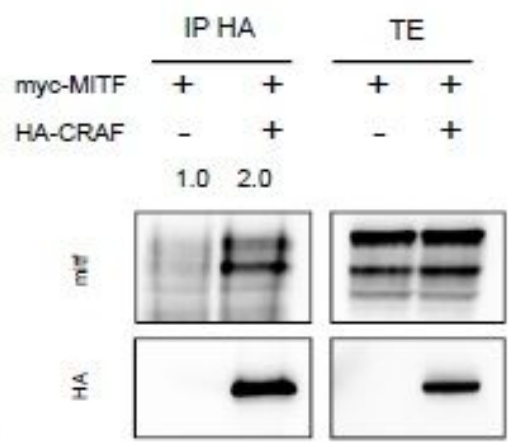

d
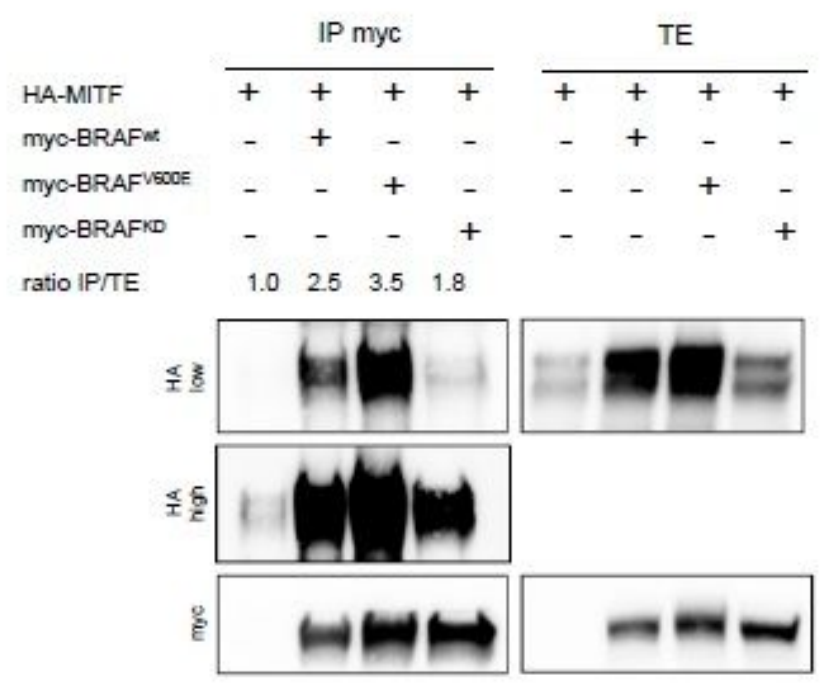

e
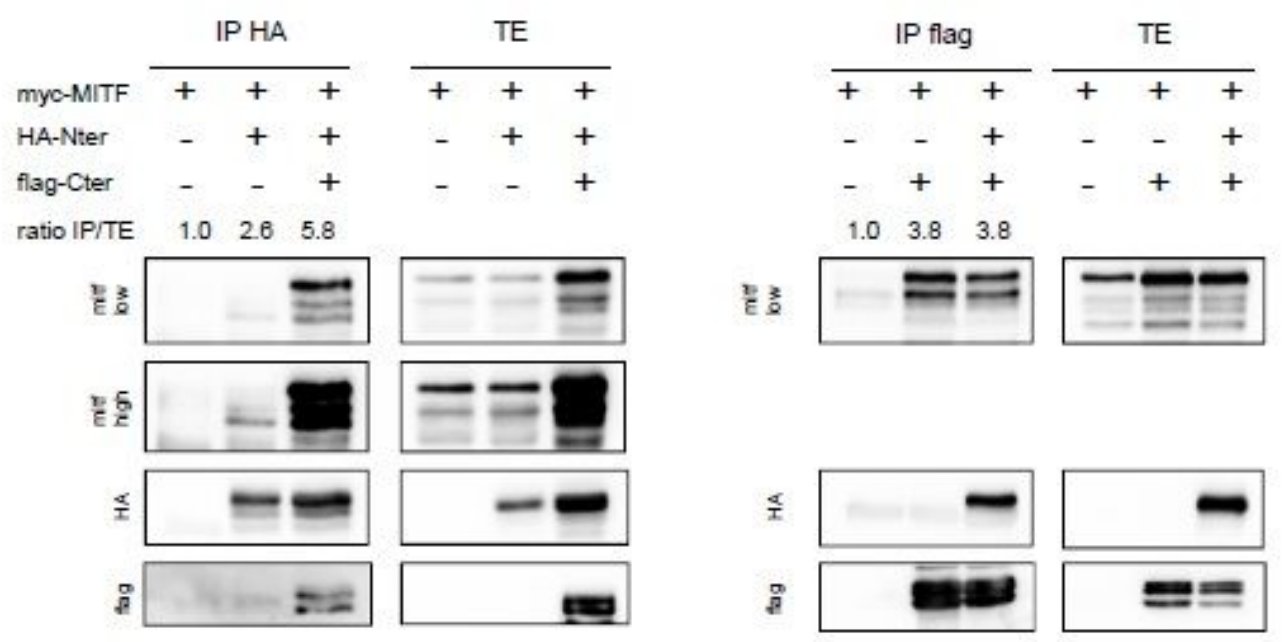

\section{Figure 3}

Characterization of RAF/MITF interaction. a, b, c MITF interaction with ARAF, BRAF or CRAF. HEK293T cells were cotransfected with the myc- MITF construct and each of the three HA-ARAF, HA-BRAF or HACRAF constructs (panels A, B, C respectively). RAF proteins were immunoprecipitated with anti-HA antibody. Immune complexes and total extracts were immunoblotted with anti-myc or anti-HA. Quantifications of the protein ratio in the immunoprecipitation over the total extract IP/TE confirmed the 
specificity of these interactions. d HEK293T cells are cotransfected with the HA-MITF construct and each of the three myc-BRAFWT, myc-BRAFV600E or myc-BRAFKD constructs. Cell lysates were immunoprecipitated with anti-myc antibody. Immune complexes and total extracts were revealed with anti-HA and anti-myc antibodies. Coimmunoprecipitations were quantified using Image $\mathrm{J}$ software by measuring signal intensity in immunoprecipitation over total extract and normalized on control lane. e HEK293T cells were cotransfected with the myc-MITF construct and HA-Nter or flag-Cter or both constructs. Cell lysates were immunoprecipitated either with anti-flag or anti-HA antibodies, and immune complexes were revealed with anti-MITF, anti-HA or anti-flag antibodies. Transfection efficiency was monitored by direct western blotting of total protein extracts. 
a

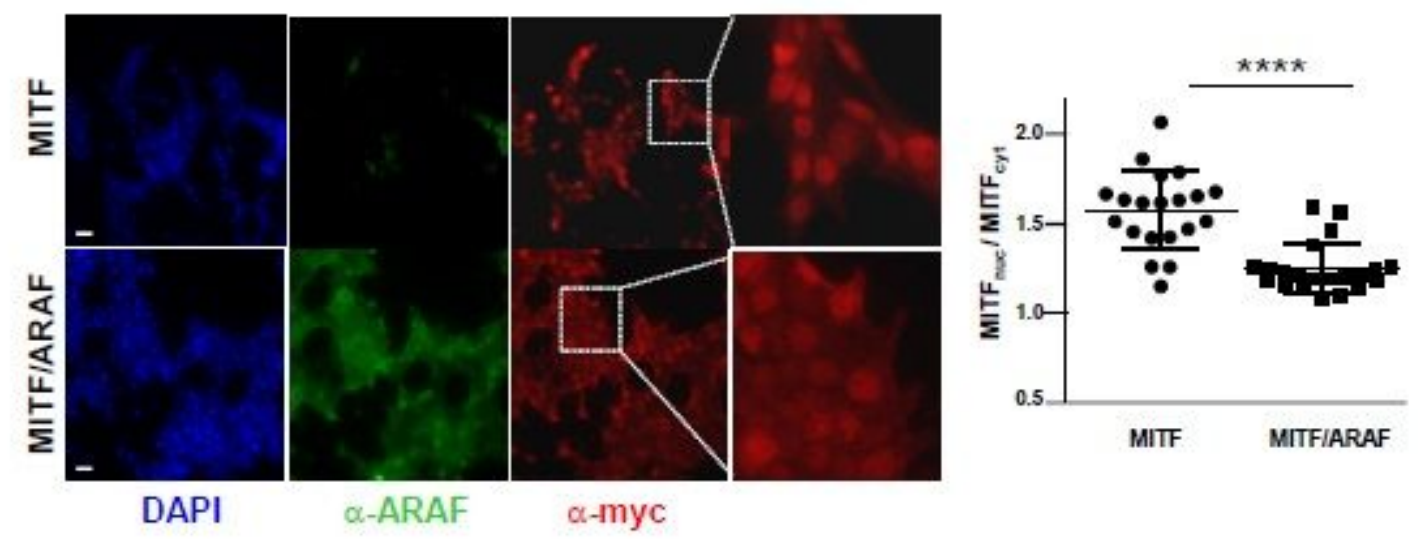

b

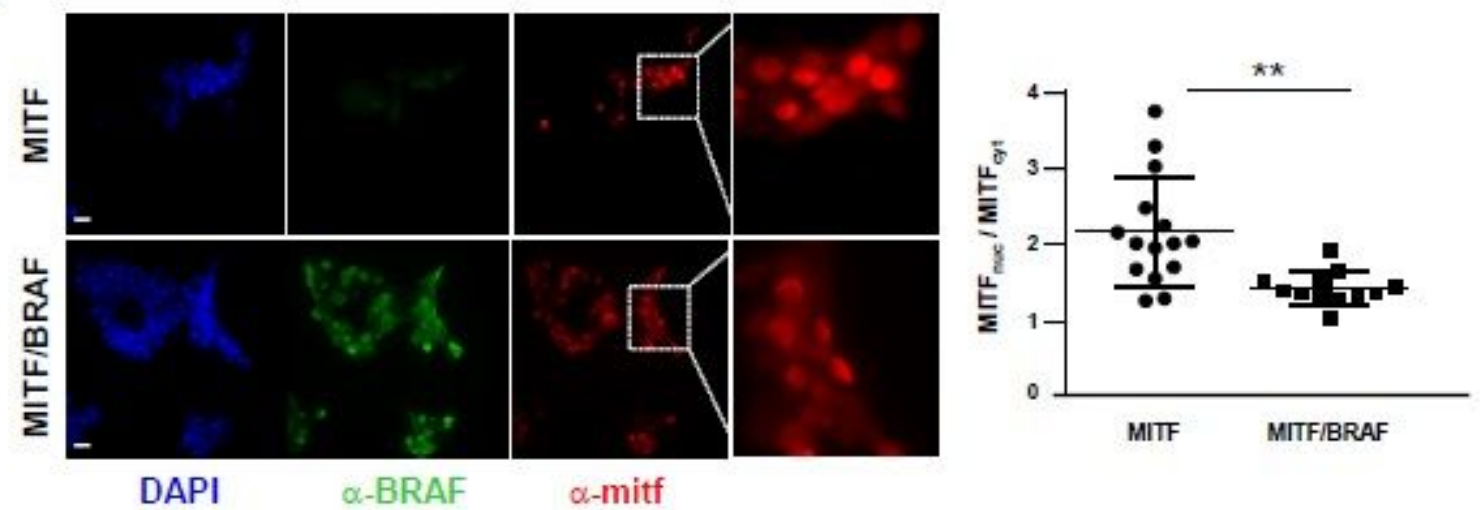

C
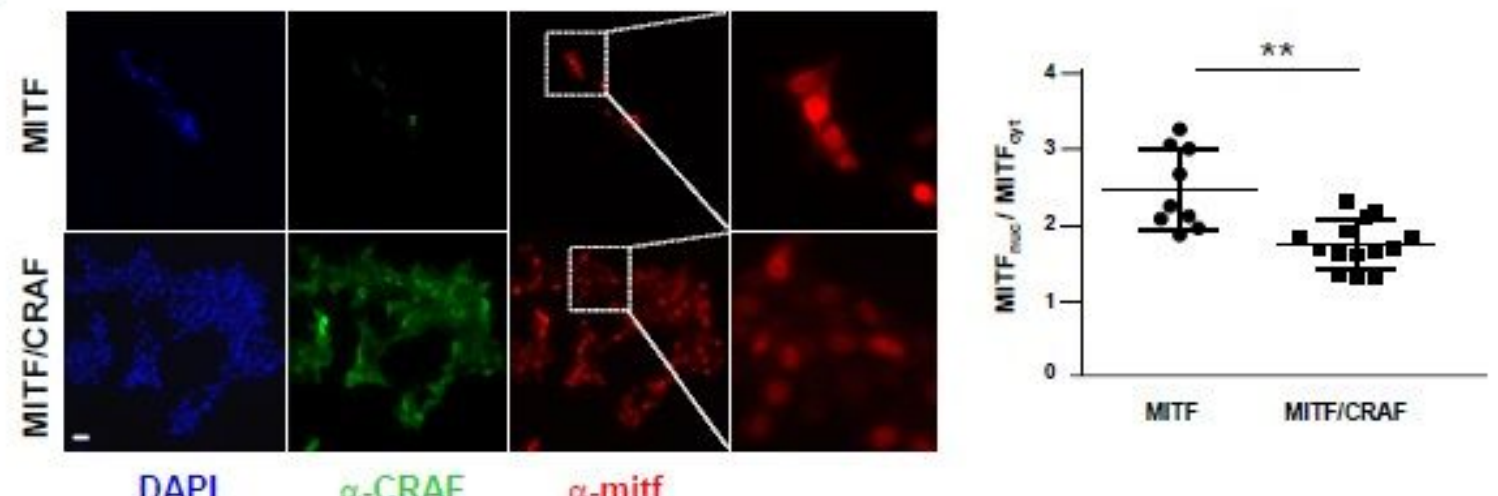

Figure 4

Effect of RAF proteins on MITF subcellular localization. Subcellular localization of MITF in the presence of ARAF (a), BRAF (b) or CRAF (c). HEK293T cells were cotransfected with myc-MITF and HA-ARAF, HABRAF or HA-CRAF. Cell immunostaining was performed with anti-myc or anti-MITF and anti-ARAF, antiBRAF or anti-CRAF. Scatter plots represent the ratio MITFnuc/MITFcyt calculated by measuring the quantity of nuclear MITF over cytoplasmic MITF. Means with standard deviations are shown. Scale bar: 
$200 \mu \mathrm{m} ;{ }^{* \star \star *}$ pvalue $<0.0001,{ }^{* \star} \mathrm{p}$-value $=0.014$ or 0.029 (for BRAF and CRAF, respectively) compared by unpaired ttest with Welch's correction.
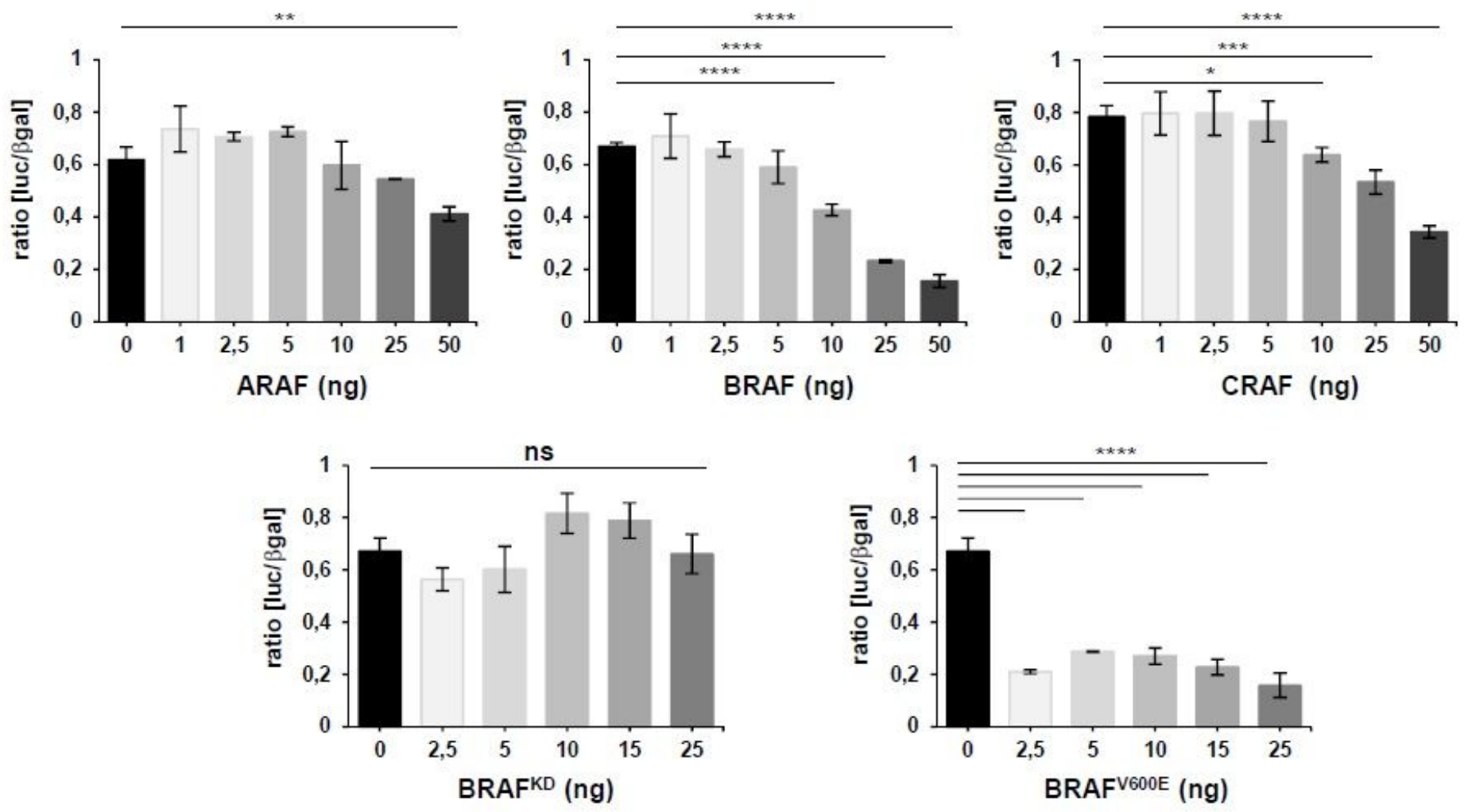

Figure 5

Effect of RAF proteins on MITF transcriptional activity. HEK293T cells were cotransfected with $5 \mathrm{ng}$ of MITF plasmid and increasing doses of either ARAF, CRAF, BRAFWT, BRAFV600E or BRAFKD constructs in the presence of a TYR-Luc luciferase reporter and a control actin galactosidase reporter. The ratio of luciferase to galactosidase activities is shown as the mean $\pm S D$ of three replicates $(n=3)$. One-way ANOVA test was performed to compare all conditions and Dunnett's tests for the multiple comparisons to

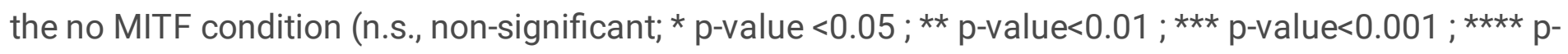
value $<0.0001)$.

\section{Supplementary Files}

This is a list of supplementary files associated with this preprint. Click to download.

- table1Estradaetal.xlsx 